\title{
Harmonic maps and strictly pseudoconvex CR manifolds
}

\author{
Robert Petit
}

\begin{abstract}
We study harmonic maps from strictly pseudoconvex $C R$ manifolds into Riemannian manifolds of nonpositive curvature. Some $C R$ analogues of the Corlette and Siu-Sampson formulas are obtained using tools of Spinorial Geometry (Dirac bundles and Dirac operators). As a main application, we obtain results about the curvature of strictly pseudoconvex $C R$ manifolds. In particular, a rigidity theorem for Sasakian manifolds is proved.
\end{abstract}

\section{Introduction.}

It is now well known that harmonic maps are a powerful tool to investigate the geometry of Riemannian manifolds. In particular, the famous rigidity results on Kähler structures with strongly negative curvature (Siu [20]) and also on the symmetric spaces (Corlette [4], Mok-Siu-Yeung [16]) have been obtained in this way.

The purpose of this article is to study harmonic maps in the framework of strictly pseudoconvex $C R$ manifolds and to deduce from that, results about the geometry of such manifolds. Remember that the strictly pseudoconvex $C R$ manifolds are abstract models of strictly pseudoconvex real hypersurfaces in complex manifolds. Standard examples are the odd-dimensional spheres and the Heisenberg groups. A strictly pseudoconvex $C R$ manifold is endowed with a natural connection called the Tanaka-Webster connection for which the complex structure, the pseudo-Hermitian structure, and the canonical metric are parallel tensors. The basic idea of this article is to derive, for any Dirac bundle over a strictly pseudoconvex $C R$ manifold, Bochner-Weitzenbock type identities for the Dirac operator defined from the Tanaka-Webster connection (Proposition 2.2 and Corollary 2.1). In the particular case of the Dirac bundle associated to a smooth map from a strictly pseudoconvex $C R$ manifold into another Riemannian manifold (cf. Paragraph 3), these formulas are the $C R$ analogues of the Corlette and Siu-Sampson formulas (Propositions 3.1 and 3.2). 
Most of our results deal with strictly pseudoconvex $C R$ manifolds for which the Tanaka-Webster connection has pseudo-Hermitian torsion zero (cf. Definition 2.2 ), i.e., Sasakian manifolds. Actually, let $M$ be such a manifold that we assume to be compact. In Paragraph 4, we prove (Theorem 4.1) that

any harmonic map from $M$ to a Riemannian manifold $N$ with nonpositive sectional curvature is trivial on the Reeb field associated to the pseudoHermitian structure.

As a consequence, we obtain that (Theorem 4.3)

$M$ admits no Riemannian metrics with nonpositive sectional curvature.

This theorem can be seen as the $C R$-analogue of the Hernandez theorem (cf. [11]).

In Paragraph 5, we consider maps from a compact Sasakian manifold of dimension $m>3$ into a Kähler manifold or a Sasakian manifold. In this case, remember that a $C R$-holomorphic map in the sense of Definitions 5.3 and 5.6 after, is always a harmonic map. In Theorems 5.3 and 5.4, we prove the $C R$-holomorphicity of harmonic maps under additional assumptions. Actually, if $\phi: M \rightarrow N$ is a harmonic map with rank $\geq 3$ and

i) if $N$ is a Kähler manifold with strongly negative curvature, then $\phi$ is $C R$-holomorphic or CR-antiholomorphic.

ii) if $N$ is a Sasakian manifold with strongly negative Tanaka-Webster curvature and if $\phi$ preserves the contact forms, then $\phi$ is a CRholomorphic isometric immersion.

These results are the $C R$-analogues of the Siu Strong rigidity Theorem.

As a consequence of Theorem 5.3, we obtain the following factorisation result (Corollary 5.1):

if $M$ is fibrated over a compact Kähler manifold $\tilde{M}$, then, any harmonic map with rank $\geq 3$ from $M$ into a Kähler manifold $N$ with strongly negative curvature, factors into a unique holomorphic map from $\tilde{M}$ into $N$.

There are many relations between harmonic maps from Kähler manifolds and holomorphic structures on vector bundles (cf. [3], [5], [19], [20]). In particular, any harmonic map from a compact Kähler manifold into a locally symmetric space of noncompact type induces a holomorphic structure on the pullback of the complexified tangent bundle of the target space. An analogue 
of this result for the compact Sasakian manifolds is the following (Theorem 5.1):

any harmonic map $\phi$ from $M$ into a locally symmetric space of noncompact type $N$, induces a holomorphic structure on $\phi^{*} T^{\mathbb{C}} N$ and $d \phi$ restricted to the holomorphic tangent bundle is a holomorphic $\phi^{*} T^{\mathbb{C}} N$-valued 1-form.

In Paragrah 6, we consider minimal (resp. $C R$-holomorphic) isometric immersions defined on a strictly pseudoconvex $C R$ manifold $M$ of dimension $2 d+1$ (not necessarily Sasakian nor compact) into a Riemannian manifold (resp. Sasakian manifold). Theorems 6.1 and 6.3 give obstructions to the existence of such immersions. Actually, we prove (Theorem 6.1) that

if $N$ is a Riemannian manifold with nonpositive complex sectional curvature and if the pseudo-Hermitian torsion satisfies at a point, $|\tau|^{2} \leq d(d-1)$, then there is no minimal isometric immersion from $M$ to $N$.

Moreover, if $M$ is not Sasakian (i.e., $\tau \neq 0$ ), we obtain that (Theorem 6.3) there is no $C R$-holomorphic isometric immersion from $M$ into a Sasakian manifold.

This last result generalizes the result of Barletta and Dragomir[1] on the non existence of $C R$-holomorphic isometric immersions from some compact quotients of the Heisenberg group into the Heisenberg group.

A last application deals with the curvature (of the Levi-Civita connection) of strictly pseudoconvex $C R$ manifolds. It is well known that the sectional curvature of a Sasakian manifold is positive on any planes containing the Reeb field. Theorem 6.2 asserts that, on any strictly pseudoconvex $C R$ manifold, there exists, at each point, a complex 2-plane for which the complex sectional curvature is positive.

The author wants to thank professors Ahmad El Soufi, Eric Loubeau, Jean-Louis Milhorat and Michel Rumin for their comments on this article.

\section{Strictly pseudoconvex CR manifolds and Dirac bundles.}

\section{Strictly pseudoconvex $C R$ manifolds.}

A smooth manifold $M$ of real dimension $m=2 d+1$ is said to be a $C R$ manifold (of $C R$ dimension $d$ ) if there exists a smooth rank $d$ complex subbundle $T^{1,0} M \subset T^{\mathbb{C}} M$ such that:

$$
T^{1,0} M \cap T^{0,1} M=\{0\}
$$


and

$$
\left[\Gamma\left(T^{1,0} M\right), \Gamma\left(T^{1,0} M\right)\right] \subset \Gamma\left(T^{1,0} M\right),
$$

where $T^{0,1} M=\overline{T^{1,0} M}$ is the complex conjuguate of $T^{1,0} M$. If $M$ is a $C R$ manifold, then its Levi distribution is the real subbundle $H$ of $T M$ defined by $H=\operatorname{Re}\left\{T^{1,0} M \oplus T^{0,1} M\right\}$. There exists on $H$, a complex structure $J$, given by $J(Z+\bar{Z})=\sqrt{-1}(Z-\bar{Z})$ for with $Z \in T^{1,0} M$.

Assume $M$ to be orientable. Then, the real line bundle $H^{\perp} \subset T^{*} M$ over $M$ admits a global nonvanishing section $\theta$. Such a section $\theta$ is called a pseudo-Hermitian structure. In this case, the Levi form $L_{\theta}$, is the Hermitian form on $H^{\mathbb{C}}=T^{1,0} M \oplus T^{0,1} M$ defined, for any $Z, W \in T^{1,0} M$, by:

$$
\begin{aligned}
& L_{\theta}(Z, W)=-\sqrt{-1} d \theta(Z, \bar{W}) \\
& L_{\theta}(\bar{Z}, \bar{W})=\overline{L_{\theta}(Z, W)} \\
& L_{\theta}(\bar{Z}, W)=L_{\theta}(Z, \bar{W})=0 .
\end{aligned}
$$

If $X, Y \in H$ are real vectors, then

$$
L_{\theta}(X, Y)=d \theta(X, J Y) .
$$

Definition 2.1. An orientable $C R$ manifold endowed with a pseudoHermitian structure is called a pseudo-Hermitian manifold. A pseudoHermitian manifold $(M, \theta)$ is said to be a strictly pseudoconvex $C R$ manifold if its Levi form $L_{\theta}$ is positive definite.

If $(M, \theta)$ is strictly pseudoconvex, then there exists a unique nonvanishing vector field $\xi$ on $M$, transverse to $H$, satisfying $\theta(\xi)=1$ and $d \theta(\xi,)=$.0 . Now, extending $J$ on $T M$ by $J \xi=0$, we can extend $L_{\theta}$ on $T M$ by the same formula as above. This allows us to define a Riemannian metric $g_{\theta}$, called the Webster metric, defined for all $X, Y \in T M$, by:

$$
g_{\theta}(X, Y)=L_{\theta}(X, Y)+\theta(X) \theta(Y) .
$$

As a consequence of the $J$-invariance of $d \theta$, we obtain that $g_{\theta}(J X, J Y)=$ $g_{\theta}(X, Y)-\theta(X) \theta(Y)$, and that, the 2-form $\omega_{\theta}$ defined by $\omega_{\theta}(X, Y)=$ $g_{\theta}(J X, Y)$ coincides with the 2 -form $d \theta$. Notice that the norm of $\omega_{\theta}$ is constant and equal to $\sqrt{d}$.

\section{Example 2.1.}

1) The odd-dimensional spheres. The odd-dimensional sphere $S^{2 d+1}$ has a standard $C R$ structure given by $T^{1,0} S^{2 d+1}=T^{1,0} \mathbb{C}^{d+1} \cap \mathbb{C} T S^{2 d+1}$. 
The pseudo-Hermitian structure is given by $i^{*} \theta$ where $i$ is the canonical injection $S^{2 d+1} \subset \mathbb{C}^{d+1}$ and where $\theta$ is the 1 -form on $\mathbb{C}^{d+1}$ given by

$$
\theta=\frac{\sqrt{-1}}{2}(\bar{\partial}-\partial)|z|^{2} \text {. }
$$

2) The Heisenberg group and its quotients.

The Heisenberg group denoted by $\mathcal{H}^{d}$ is obtained as $\mathbb{C}^{d} \times \mathbb{R}$ with the group law

$$
(z, t) \cdot(w, s)=(z+w, t+s+2 \operatorname{Im}\langle z, w\rangle),
$$

where $\langle$,$\rangle is the Hermitian product (cf. Dragomir [6]). The C R$ structure is given by $T^{1,0} \mathcal{H}^{d}=\sum_{j \leq d} \mathbb{C} Z_{j}$, where $Z_{j}=\frac{\partial}{\partial z_{j}}+\sqrt{-1} \overline{z_{j}} \frac{\partial}{\partial t}$ and $\frac{\partial}{\partial z_{j}}=\frac{1}{2}\left(\frac{\partial}{\partial x_{j}}-\sqrt{-1} \frac{\partial}{\partial y_{j}}\right)$, with $z_{j}=x_{j}+\sqrt{-1} y_{j}$. The pseudoHermitian structure is given by

$$
\theta_{0}=d t+2 \sum_{j \leq d}\left(x_{j} d y_{j}-y_{j} d x_{j}\right) .
$$

Let $\delta_{s}: \mathcal{H}^{d}-\{0\} \rightarrow \mathcal{H}^{d}-\{0\}, s>0$, be the dilatation defined by $\delta_{s}(z, t)=\left(s z, s^{2} t\right)$. For $m \in \mathbb{N}^{*}$, we set $\delta_{s}^{m}=\delta_{s} \circ \ldots \circ \delta_{s}(m$ factors) and $\delta_{s}^{-m}=\delta_{\frac{1}{s}}^{m}$. For $d>1$ and $0<s<1$, the discrete group $G_{s}=\left\{\delta_{s}^{m}, m \in Z\right\}$ acts freely on $\mathcal{H}^{d}-\{0\}$ as a properly discontinuous group of $C R$ automorphisms of $\mathcal{H}^{d}-\{0\}$. The quotient space $\mathcal{H}^{d}(s)=\left(\mathcal{H}^{d}-\{0\}\right) / G_{s}$ (cf. Dragomir[7]) is a compact strictly pseudoconvex $C R$ manifold diffeomorphic to $\Sigma^{2 d} \times S^{1}$, where $\Sigma^{2 d}=$ $\left\{x \in \mathcal{H}^{d},|x|=1\right\}$ and $|x|=\left(|z|^{4}+t^{2}\right)^{\frac{1}{4}}$ is the Heisenberg norm of $x=(z, t)$. Let $\pi: \mathcal{H}^{d}-\{0\} \rightarrow \mathcal{H}^{d}(s)$ be the natural covering map. Then the pseudo-Hermitian structure is given by

$$
\theta(\pi(x))=|x|^{-2} \theta_{0}(x) \circ(d \pi(x))^{-1},
$$

with $x \in \mathcal{H}^{d}-\{0\}$.

An other example of compact strictly pseudoconvex $C R$ manifold obtained as a quotient of $\mathcal{H}^{d}$ by a discrete group is the Heisenberg nilmanifold (cf. Urakawa[23]).

3) Other examples.

Remember that the Siegel domains are domains of $\mathbb{C}^{d+1}$ defined by:

$$
D_{\alpha, \beta}=\left\{\left(z_{1}, \ldots, z_{d}, z_{d+1}\right) \in \mathbb{C}^{d+1}: \sum_{1 \leq j \leq d}\left|z_{j}\right|^{2 \alpha_{j}}+\operatorname{Im}\left(z_{d+1}^{\beta}\right)-1<0\right\},
$$


with $(\alpha, \beta)=\left(\alpha_{1}, \ldots, \alpha_{d}, \beta\right) \in Z_{+}^{d+1}$. The boundaries of these domains, called Pseudo-Siegel domains, are strictly pseudoconvex $C R$ manifolds (cf. [1]). Note that the Heisenberg group is diffeomorphic to the boundary of $D_{1,1}$. Other examples are given by the unit tangent bundle over a constant curved manifold (cf. [22]) or the total space of the Boothby-Wang fibration over a compact Hodge manifold.

On a strictly pseudoconvex $C R$ manifold, there exists a canonical connection preserving together the complex structure of the Levi distribution, the pseudo-Hermitian structure and the Webster metric. Actually

Proposition 2.1 (Tanaka-Webster connection cf. [21], [25]). Let $\left(M, \theta, \xi, J, g_{\theta}\right)$ be a strictly pseudoconvex $C R$ manifold, then there exists a unique affine connection $\nabla$ on TM (called the Tanaka-Webster connection) such that:

a) The distribution $H$ is parallel for $\nabla$.

b) $\nabla g_{\theta}=0, \nabla J=0, \nabla \theta=0$ (hence $\left.\nabla \xi=\nabla \omega_{\theta}=0\right)$.

c) The torsion $T$ of $\nabla$ satisfies for any $X, Y \in H, T(X, Y)=-\omega_{\theta}(X, Y) \xi$ and $T(\xi, J X)=-J T(\xi, X)$.

Note that, unlike the Levi-Civita connection, the torsion of the TanakaWebster connection is always non zero.

The pseudo-Hermitian torsion, denoted $\tau$, is the $T M$-valued 1-form defined by $\tau(X)=T(\xi, X)$. Note that $\tau$ is $g_{\theta}$-symmetric and trace-free.

Definition 2.2. A strictly pseudoconvex $C R$ manifold is called a normal strictly pseudoconvex $C R$ manifold or a Sasakian manifold if the pseudoHermitian torsion is zero.

Pseudo-Siegel domains (in particular the Heisenberg group) are Sasakian manifolds. The compact regular strictly pseudoconvex $C R$ manifolds, such as the odd dimensional spheres or the Heisenberg nilmanifold, are examples of compact Sasakian manifolds. On the contrary, the manifolds $\mathcal{H}^{d}(s)$ are examples of compact strictly pseudoconvex $C R$ manifolds which are not Sasakian (the calculation of the pseudo-Hermitian torsion associated to $\theta=$ $f \theta_{0}$ is obtained using formula (2.16) in [15, p. 164]).

The curvature of the Tanaka-Webster connection $\nabla$, denoted $R$, is given for any $X, Y \in T M$ by

$$
R(X, Y)=\nabla_{Y} \nabla_{X}-\nabla_{X} \nabla_{Y}+\nabla_{[X, Y]}
$$


The Bianchi identities are the following (cf. [21], [10], [17]):

$$
\begin{aligned}
& \qquad \begin{aligned}
R(X, Y) Z+R(Z, X) Y+R(Y, Z) X= & \omega_{\theta}(X, Y) \tau(Z)+\omega_{\theta}(Z, X) \tau(Y) \\
& +\omega_{\theta}(Y, Z) \tau(X)
\end{aligned} \\
& \qquad \begin{aligned}
R(X, \xi) Z+R(\xi, Z) X=\left(\nabla_{X} \tau\right)(Z)- & \left(\nabla_{Z} \tau\right)(X) \quad(X, Y, Z \in H),
\end{aligned} \\
& \text { where, }\left(\nabla_{X} \tau\right)(Z)=\nabla_{X} \tau(Z)-\tau\left(\nabla_{X} Z\right) .
\end{aligned}
$$

The Ricci endomorphism Ric is defined by:

$$
\operatorname{Ric}(X)=\sum_{i} R\left(e_{i}, X\right) e_{i},
$$

where $\left\{e_{i}\right\}$ is a $g_{\theta}$-orthonormal basis.

Note that $\operatorname{Ric}(\xi)=\delta \tau$ with $\delta \tau=-\sum_{i}\left(\nabla_{e_{i}} \tau\right)\left(e_{i}\right)$. Unlike the Kähler case, $R$ and $R i c$ are not in general $J$-invariant. Nevertheless, we have the identities:

$R(X, Y)-R(J X, J Y)=J \tau(X) \wedge Y-J \tau(Y) \wedge X-\tau(X) \wedge J Y+\tau(Y) \wedge J X$

$$
\operatorname{Ric}(J X)-J \operatorname{Ric}(X)=2(d-1) \tau(X) \quad(X, Y \in H) .
$$

\section{Dirac bundles and Dirac operators over strictly pseudoconvex $C R$ manifolds.}

In this paragraph, all the canonical connections will be denoted by $\nabla$.

Let $M$ be a strictly pseudoconvex $C R$ manifold and $T M$, its tangent bundle. Endowed with the Webster metric and the Tanaka-Webster connection, the bundle $T M$ is an oriented Riemannian vector bundle with a Riemannian connection. Since $T M$ is an oriented vector bundle, then the Clifford bundle $C l(M)$ is well defined (Definition 3.4 Chapter II of [14]). The Webster metric and the Tanaka-Webster connection extend from $T M$ to $C l(M)$ in such a way that the induced connection on $C l(M)$ be a Riemannian connection acting as a derivation on $C l(M)$. Now, let $S$ be a vector bundle over $M$ of left modules over $C l(M)$, endowed with a Riemannian metric and a Riemannian connection. In addition, we assume that the unit vectors in $T M$ act isometrically on $S$ and that the connection on $S$ is a module derivative. Then, under the previous assumptions, $S$ is called a Dirac 
bundle (cf. [14] for more details). Let $S$ be such a bundle over $M$, then the canonical Dirac operator $\mathcal{D}$ acting on $\Gamma(S)$ is given by:

$$
\mathcal{D}=\sum_{i} e_{i} \cdot \nabla_{e_{i}}
$$

where $\left\{e_{i}\right\}$ is a local orthonormal tangent frame.

In this context, we define two other differential operators on $\Gamma(S)$ (which will play an important role later on). The first, associated with $J$ and denoted by $\mathcal{D}_{J}$ is defined by:

$$
\mathcal{D}_{J}=\sum_{i} e_{i} \cdot \nabla_{J e_{i}} \cdot
$$

The second, associated with $\tau$ and denoted by $\mathcal{D}_{\tau}$ is:

$$
\mathcal{D}_{\tau}=\sum_{i} e_{i} \cdot \nabla_{\tau\left(e_{i}\right)} .
$$

The operator $\mathcal{D}_{J}$ (resp. $\mathcal{D}_{\tau}$ ) will be called the $J$-twisted Dirac operator (resp. the $\tau$-twisted Dirac operator). In the following, we denote by $\lambda$. the endomorphism of $\Gamma(S)$ given by left module multiplication by a form or a vector field and by $[$,$] (resp. \{$,$\} ) the commutator (resp. anticommutator).$

Lemma 2.1. The operators $\mathcal{D}, \mathcal{D}_{J}$ and $\mathcal{D}_{\tau}$ satisfy the following identities:

$$
\begin{aligned}
\left\{\mathcal{D}, \lambda_{\xi}\right\} & =-2 \nabla_{\xi} \\
\left\{\mathcal{D}_{\tau}, \lambda_{\xi}\right\} & =0 \\
{\left[\mathcal{D}, \lambda_{\omega_{\theta}}\right] } & =2 \mathcal{D}_{J} .
\end{aligned}
$$

Proof. Since $\xi$ is parallel for the Tanaka-Webster connection, the endomorphism $\lambda_{\xi}$ is parallel (i.e., $\left[\nabla, \lambda_{\xi}\right]=0$ ). We deduce that

$$
\begin{aligned}
\left\{\mathcal{D}, \lambda_{\xi}\right\} & =\mathcal{D} \circ \lambda_{\xi}+\lambda_{\xi} \circ \mathcal{D}=\sum_{i}\left(e_{i} \cdot \nabla_{e_{i}} \circ \lambda_{\xi}+\xi \cdot e_{i} \cdot \nabla_{e_{i}}\right) \\
& =\sum_{i}\left(e_{i} \cdot \xi+\xi \cdot e_{i}\right) \cdot \nabla_{e_{i}}=-2 \sum_{i} \theta\left(e_{i}\right) \nabla_{e_{i}}=-2 \nabla_{\xi},
\end{aligned}
$$

and

$$
\left\{\mathcal{D}_{\tau}, \lambda_{\xi}\right\}=\mathcal{D}_{\tau} \circ \lambda_{\xi}+\lambda_{\xi} \circ \mathcal{D}_{\tau}=\sum_{i}\left(e_{i} \cdot \xi+\xi \cdot e_{i}\right) \cdot \nabla_{\tau\left(e_{i}\right)}=-2 \sum_{i} \nabla_{\tau(\xi)}=0
$$


Since $\omega_{\theta}$ is also parallel, $\left[\nabla, \lambda_{\omega_{\theta}}\right]=0$ and therefore

$$
\begin{aligned}
{\left[\mathcal{D}, \lambda_{\omega_{\theta}}\right] } & =\mathcal{D} \circ \lambda_{\omega_{\theta}}-\lambda_{\omega_{\theta}} \circ \mathcal{D}=\sum_{i}\left(e_{i} \cdot \omega_{\theta}-\omega_{\theta} \cdot e_{i}\right) \cdot \nabla_{e_{i}} \\
& =-2 \sum_{i} J e_{i} \cdot \nabla_{e_{i}}=2 \mathcal{D}_{J}
\end{aligned}
$$

Let $E$ be a Riemannian vector bundle over $M$ and $\alpha$ be a $E$-valued $p$-form on $M$. Setting $\alpha_{H}=\alpha \circ \Pi$ where $\Pi: T M \rightarrow H$ is the canonical projection, we have (cf. [18]) the decomposition $\alpha=\alpha_{H}+\theta \wedge i(\xi) \alpha(\wedge$ denotes the exterior product). Moreover, under the $J$-action, we have $\alpha_{H}=\alpha_{H}^{+}+\alpha_{H}^{-}$ with $\alpha_{H}^{+}$(respectively $\alpha_{H}^{-}$) is the $J$-anti-invariant part of $\alpha_{H}$ (respectively the $J$-invariant part of $\alpha_{H}$ ) (i.e. $\alpha_{H}^{ \pm}=\frac{1}{2}\left(\alpha_{H} \pm \alpha_{H} \circ J\right)$ ). In particular, we have $T=T_{H}^{+}+\theta \wedge \tau$, with $T_{H}^{+}=-\omega_{\theta} \otimes \xi$.

In the following, $\nabla^{2}$. denotes the second covariant derivative on $\Gamma(S)$ (i.e., $\nabla_{X, Y}^{2}=\nabla_{X} \nabla_{Y}-\ddot{\nabla}_{\nabla_{X} Y}$ ) and $\nabla^{*} \nabla$ the rough Laplacian (i.e., $\nabla^{*} \nabla=$ $\left.-\operatorname{trace}_{g_{\theta}} \nabla_{., .}^{2}\right)$.

Proposition 2.2 (Weitzenbock formulas). The operators $\mathcal{D}^{2}$ and $\mathcal{D}_{J}^{2}$ satisfy the relations:

$$
\begin{gathered}
\mathcal{D}^{2}=\nabla^{*} \nabla-\lambda_{\omega_{\theta}} \circ \nabla_{\xi}+\mathcal{R}_{H}+\lambda_{\xi} \circ\left(\mathcal{D}_{\tau}-2 \lambda_{\xi} \circ \mathcal{R}_{\xi}\right) \\
\mathcal{D}_{J}^{2}-\nabla_{\xi, \xi}^{2}=\nabla^{*} \nabla-\lambda_{\omega_{\theta}} \circ \nabla_{\xi}+\mathcal{R}_{H}^{+}-\mathcal{R}_{H}^{-}
\end{gathered}
$$

where $\mathcal{R}_{H}, \mathcal{R}_{H}^{ \pm}$and $\mathcal{R}_{\xi}$ are the endomorphisms given respectively by:

$$
\begin{gathered}
\mathcal{R}_{H}=-\frac{1}{2} \sum_{i, j} e_{i} \cdot e_{j} \cdot R_{H}\left(e_{i}, e_{j}\right), \quad \mathcal{R}_{H}^{ \pm}=-\frac{1}{2} \sum_{i, j} e_{i} \cdot e_{j} \cdot R_{H}^{ \pm}\left(e_{i}, e_{j}\right), \\
\mathcal{R}_{\xi}=-\frac{1}{2} \sum_{i} \xi \cdot e_{i} \cdot R\left(\xi, e_{i}\right) .
\end{gathered}
$$

Proof. In a local orthonormal tangent frame, we have:

$$
\begin{aligned}
\mathcal{D}^{2} & =\sum_{i, j} e_{i} \cdot \nabla_{e_{i}}\left(e_{j} \cdot \nabla_{e_{j}}\right)=\sum_{i, j}\left(e_{i} \cdot\left(\nabla_{e_{i}} e_{j}\right) \cdot \nabla_{e_{j}}+e_{i} \cdot e_{j} \cdot \nabla_{e_{i}} \nabla_{e_{j}}\right) \\
& =\sum_{i, j} e_{i} \cdot e_{j} \cdot\left(\nabla_{e_{i}} \nabla_{e_{j}}-\nabla_{e_{i} e_{j}}\right)=\sum_{i, j} e_{i} \cdot e_{j} \cdot \nabla_{e_{i}, e_{j}}^{2} \\
& =-\sum_{i} \nabla_{e_{i}, e_{i}}^{2}-\frac{1}{2} \sum_{i, j} e_{i} \cdot e_{j} \cdot\left(\nabla_{e_{j}, e_{i}}^{2}-\nabla_{e_{i}, e_{j}}^{2}\right) .
\end{aligned}
$$


Since $\nabla_{Y, X}^{2}-\nabla_{X, Y}^{2}=R(X, Y)-\nabla_{T(X, Y)}$ and $R=R_{H}+\theta \wedge i(\xi) R$, we deduce that

$$
\begin{aligned}
\nabla_{Y, X}^{2}-\nabla_{X, Y}^{2}= & R_{H}(X, Y)+\omega_{\theta}(X, Y) \nabla_{\xi}+\theta(X)\left(R(\xi, Y)-\nabla_{\tau(Y)}\right) \\
& -\theta(Y)\left(R(\xi, X)-\nabla_{\tau(X)}\right)
\end{aligned}
$$

Moreover, we have $\xi=\sum_{i} \theta\left(e_{i}\right) e_{i}$ and, by the isomorphism $\wedge^{*} M \simeq C l(M)$, $\omega_{\theta}=\frac{1}{2} \sum_{i, j} \omega_{\theta}\left(e_{i}, e_{j}\right) e_{i} . e_{j}$. Hence $(4)$ becomes:

$$
\begin{aligned}
\mathcal{D}^{2}=-\sum_{i} \nabla_{e_{i}, e_{i}}^{2}-\omega_{\theta} \cdot \nabla_{\xi}- & \frac{1}{2} \sum_{i, j} e_{i} \cdot e_{j} \cdot R_{H}\left(e_{i}, e_{j}\right) \\
& -\frac{1}{2} \sum_{i}\left(\xi \cdot e_{i}-e_{i} \cdot \xi\right) \cdot\left(R\left(\xi, e_{i}\right)-\nabla_{\tau\left(e_{i}\right)}\right) .
\end{aligned}
$$

Using the relations $e_{i} \cdot \xi=-\xi . e_{i}-2 g_{\theta}\left(\xi, e_{i}\right)$ and $\tau(\xi)=0$, we obtain the formula.

We have for $\mathcal{D}_{J}^{2}$ :

$$
\begin{aligned}
\mathcal{D}_{J}^{2} & =\sum_{i, j}\left(e_{i} \cdot\left(\nabla_{J e_{i}} e_{j}\right) \cdot \nabla_{J e_{j}}+e_{i} \cdot e_{j} \cdot \nabla_{J e_{i}} \nabla_{J e_{j}}\right) \\
& =\sum_{i, j} e_{i} \cdot e_{j} \cdot\left(\nabla_{J e_{i}} \nabla_{J e_{j}}-\nabla_{J \nabla_{J e_{i}} e_{j}}\right) .
\end{aligned}
$$

Since $\nabla J=0,(6)$ becomes:

$$
\begin{aligned}
\mathcal{D}_{J}^{2} & =\sum_{i, j} e_{i} \cdot e_{j} \cdot\left(\nabla_{J e_{i}} \nabla_{J e_{j}}-\nabla_{\nabla_{J e_{i}} J e_{j}}\right)=\sum_{i, j} e_{i} \cdot e_{j} \cdot \nabla_{J e_{i}, J e_{j}}^{2} \\
& =-\sum_{i} \nabla_{J e_{i}, J e_{i}}^{2}-\frac{1}{2} \sum_{i, j} e_{i} \cdot e_{j} \cdot\left(\nabla_{J e_{j}, J e_{i}}^{2}-\nabla_{J e_{i}, J e_{j}}^{2}\right) .
\end{aligned}
$$

Using, on the one hand, the identities $\theta \circ J=0$ and $\omega_{\theta} \circ J=\omega_{\theta}$, and, on the other hand, $R \circ J=R_{H} \circ J=R_{H}^{+} \circ J+R_{H}^{-} \circ J=R_{H}^{+}-R_{H}^{-}$, we obtain from (5):

$$
\mathcal{D}_{J}^{2}=-\sum_{i} \nabla_{J e_{i}, J e_{i}}^{2}-\omega_{\theta} \cdot \nabla_{\xi}-\frac{1}{2} \sum_{i, j} e_{i} \cdot e_{j} \cdot R_{H}^{+}\left(e_{i}, e_{j}\right)+\frac{1}{2} \sum_{i, j} e_{i} \cdot e_{j} \cdot R_{H}^{-}\left(e_{i}, e_{j}\right)
$$


Now, in a local orthonormal tangent frame $\left\{\epsilon_{1}, \ldots \epsilon_{d}, J \epsilon_{1}, \ldots J \epsilon_{d}, \xi\right\}$, where $\left\{\epsilon_{1}, \ldots \epsilon_{d}, J \epsilon_{1}, \ldots J \epsilon_{d}\right\}$ is a local orthonormal frame of $H$, we have:

$$
\nabla^{*} \nabla=-\sum_{i \leq d} \nabla_{\epsilon_{i}, \epsilon_{i}}^{2}-\sum_{i \leq d} \nabla_{J \epsilon_{i}, J \epsilon_{i}}^{2}-\nabla_{\xi, \xi}^{2}
$$

Hence,

$$
\begin{aligned}
-\sum_{i \leq m} \nabla_{J e_{i}, J e_{i}}^{2} & =-\sum_{i \leq d} \nabla_{J \epsilon_{i}, J \epsilon_{i}}^{2}-\sum_{i \leq d} \nabla_{\epsilon_{i}, \epsilon_{i}}^{2} \\
& =\nabla^{*} \nabla+\nabla_{\xi, \xi}^{2}
\end{aligned}
$$

This concludes the proof of the second identity.

In the following, a local orthonormal tangent frame $\left\{\epsilon_{1}, \ldots, \epsilon_{d}, J \epsilon_{1}, \ldots\right.$, $\left.J \epsilon_{d}, \xi\right\}$, where $\left\{\epsilon_{1}, \ldots \epsilon_{d}, J \epsilon_{1}, \ldots J \epsilon_{d}\right\}$ is a local orthonormal frame of $H$, will be called an adapted frame.

Corollary 2.1. The following identities hold:

(8) $\quad-\frac{1}{2} \lambda_{\xi} \circ\left\{\mathcal{D}^{2}, \lambda_{\xi}\right\}=\nabla^{*} \nabla-\lambda_{\omega_{\theta}} \circ \nabla_{\xi}+\mathcal{R}_{H}=\mathcal{D}_{J}^{2}-\nabla_{\xi, \xi}^{2}+2 \mathcal{R}_{H}^{-}$.

Proof. We have

$\mathcal{D}^{2}=\frac{1}{2}\left(\mathcal{D}^{2}+\lambda_{\xi} \circ \mathcal{D}^{2} \circ \lambda_{\xi}\right)+\frac{1}{2}\left(\mathcal{D}^{2}-\lambda_{\xi} \circ \mathcal{D}^{2} \circ \lambda_{\xi}\right)=\frac{1}{2} \lambda_{\xi} \circ\left[\mathcal{D}^{2}, \lambda_{\xi}\right]-\frac{1}{2} \lambda_{\xi} \circ\left\{\mathcal{D}^{2}, \lambda_{\xi}\right\}$,

where $\lambda_{\xi} \circ\left[\mathcal{D}^{2}, \lambda_{\xi}\right]$ (resp. $\left.\lambda_{\xi} \circ\left\{\mathcal{D}^{2}, \lambda_{\xi}\right\}\right)$ anticommutes (resp. commutes) with $\lambda_{\xi}$. Since $\left[R(X, Y), \lambda_{\xi}\right]=0$, we have $\left[\mathcal{R}_{H}, \lambda_{\xi}\right]=0$ and $\left\{\mathcal{R}_{\xi}, \lambda_{\xi}\right\}=0$. Now, since $\left[\nabla, \lambda_{\xi}\right]=0,\left[\lambda_{\omega_{\theta}}, \lambda_{\xi}\right]=0$ and $\left\{\mathcal{D}_{\tau}, \lambda_{\xi}\right\}=0$, we deduce that the first three terms in the right-hand side of (3) commute with $\lambda_{\xi}$, meanwhile the last anticommutes with $\lambda_{\xi}$. By identification, we obtain

$$
\frac{1}{2} \lambda_{\xi} \circ\left[\mathcal{D}^{2}, \lambda_{\xi}\right]=\lambda_{\xi} \circ\left(\mathcal{D}_{\tau}-2 \lambda_{\xi} \circ \mathcal{R}_{\xi}\right)
$$

and

$$
-\frac{1}{2} \lambda_{\xi} \circ\left\{\mathcal{D}^{2}, \lambda_{\xi}\right\}=\nabla^{*} \nabla-\lambda_{\omega_{\theta}} \circ \nabla_{\xi}+\mathcal{R}_{H}
$$

The last equality in (8) follows from the Weitzenbock formula for $\mathcal{D}_{J}^{2}$.

Under the assumption that $M$ is compact, the operators $\mathcal{D}$ and $\mathcal{D}_{J}$ have the following property: 
Proposition 2.1. The operators $\mathcal{D}$ and $\mathcal{D}_{J}$ are formally self-adjoint for the natural inner product on $\Gamma(S)$ given by:

$$
(,)=\int_{M}\langle,\rangle v_{g_{\theta}}
$$

where $v_{g_{\theta}}$ is the canonical volume element (i.e., $\left.v_{g_{\theta}}=\theta \wedge(d \theta)^{d}\right)$.

Before the proof of this proposition, remember (cf. [23]) that for any $E$-valued $p$-form $\alpha$, the covariant derivative and the exterior derivative of $\alpha$ are given by:

$$
\begin{aligned}
\left(\nabla_{X} \alpha\right)\left(X_{1}, \ldots, X_{p}\right)= & \nabla_{X}^{E} \alpha\left(X_{1}, \ldots, X_{p}\right) \\
& +\sum_{i=1}^{p} \alpha\left(X_{1}, \ldots, \nabla_{X} X_{i}, \ldots, X_{p}\right) \\
(d \alpha)\left(X_{1}, \ldots, X_{p+1}\right)= & \sum_{i=1}^{p+1}(-1)^{i+1}\left(\nabla_{X_{i}} \alpha\right)\left(X_{1}, \ldots, \hat{X}_{i}, \ldots, X_{p+1}\right) \\
& +\left(Q_{T} \alpha\right)\left(X_{1}, \ldots, X_{p+1}\right)
\end{aligned}
$$

where $\hat{X}_{i}$ means that the term $X_{i}$ is omitted and where $Q_{T}$ is the operator

$$
\begin{aligned}
& \left(Q_{T} \alpha\right)\left(X_{1}, \ldots, X_{p+1}\right) \\
& =\sum_{i\langle j}(-1)^{i+j} \alpha\left(T\left(X_{i}, X_{j}\right), X_{1}, \ldots, \hat{X}_{i}, \ldots, \hat{X}_{j}, \ldots, X_{p+1}\right) .
\end{aligned}
$$

The divergence of $\alpha$ is defined by:

$$
(\delta \alpha)\left(X_{1}, \ldots, X_{p-1}\right)=- \text { trace }_{g_{\theta}}(\nabla . \alpha)\left(., X_{1}, \ldots, X_{p-1}\right) .
$$

Note that $\delta$ is not the formal adjoint of $d$.

Proof. Let $\sigma_{1}, \sigma_{2} \in \Gamma(S)$, then (cf. [14, p. 115])

$$
\left\langle\mathcal{D} \sigma_{1}, \sigma_{2}\right\rangle v_{g_{\theta}}-\left\langle\sigma_{1}, \mathcal{D} \sigma_{2}\right\rangle v_{g_{\theta}}=\delta \alpha v_{g_{\theta}},
$$

where $\alpha$ is the 1 -form given by $\alpha(X)=\left\langle\sigma_{1}, X . \sigma_{2}\right\rangle$. Using $\nabla v_{g_{\theta}}=0$ and (10), we have, with respect to an orthonormal tangent frame $\left\{e_{i}\right\}$ :

$$
\delta \alpha v_{g_{\theta}}\left(e_{1}, \ldots, e_{m}\right)=-\sum_{i=1}^{p+1}(-1)^{i+1}\left(\nabla_{e_{i}} i\left(\alpha^{\sharp}\right) v_{g_{\theta}}\right)\left(e_{1}, \ldots, \hat{e_{i}}, \ldots, e_{m}\right),
$$


where $\alpha^{\sharp}$ is the vector field canonically associated to $\alpha$. So, using (9), we obtain

$$
\delta \alpha v_{g_{\theta}}\left(e_{1}, \ldots, e_{m}\right)=\left(-d i\left(\alpha^{\sharp}\right) v_{g_{\theta}}+Q_{T}\left(i\left(\alpha^{\sharp}\right) v_{g_{\theta}}\right)\right)\left(e_{1}, \ldots, e_{m}\right) .
$$

Now, in a local orthonormal tangent frame $\left\{f_{1}, \ldots f_{2 d}, \xi\right\}$, where $\left\{f_{i}\right\}, i \leq 2 d$ is a local orthonormal frame of $H$, we have:

$$
\begin{aligned}
\left(Q_{T} i\left(\alpha^{\sharp}\right) v_{g_{\theta}}\right)\left(f_{1}, \ldots, f_{2 d}, \xi\right) & \\
= & \sum_{i\langle j \leq 2 d}(-1)^{i+j+1} \omega_{\theta}\left(f_{i}, f_{j}\right) v_{g_{\theta}}\left(\alpha^{\sharp}, \xi, f_{1}, \ldots, \hat{f}_{i}, \ldots, \hat{f}_{j}, \ldots, f_{2 d}, \xi\right) \\
& +\sum_{i \leq 2 d}(-1)^{i} v_{g_{\theta}}\left(\alpha^{\sharp}, \tau\left(f_{i}\right), f_{1}, \ldots, \hat{f}_{i}, \ldots, f_{2 d}\right) \\
= & \alpha(\xi) \sum_{i, j \leq 2 d}(-1)^{i} g_{\theta}\left(\tau\left(f_{i}\right), f_{j}\right) v_{g_{\theta}}\left(\xi, f_{j}, f_{1}, \ldots, \hat{f}_{i}, \ldots, f_{2 d}\right) \\
= & \alpha(\xi) \sum_{i \leq 2 d}(-1)^{i} g_{\theta}\left(\tau\left(f_{i}\right), f_{i}\right) v_{g_{\theta}}\left(\xi, f_{i}, f_{1}, \ldots, \hat{f}_{i}, \ldots, f_{2 d}\right) \\
= & -\alpha(\xi)\left(\operatorname{trace}_{g_{\theta}} \tau\right) v_{g_{\theta}}\left(f_{1}, \ldots, f_{2 d}, \xi\right)=0 .
\end{aligned}
$$

Hence, $\delta \alpha v_{g_{\theta}}=-d\left(i\left(\alpha^{\sharp}\right) v_{g_{\theta}}\right)$. The result immediately follows from the Stokes formula. Since $\mathcal{D}_{J}=\frac{1}{2}\left[\mathcal{D}, \lambda_{\omega_{\theta}}\right]$, the result for $\mathcal{D}_{J}$ is deduced from the previous one.

\section{Corlette and Siu-Sampson type formulas.}

In this section, we use the previous identities to obtain Corlette and SiuSampson type formulas for maps from strictly pseudoconvex $C R$ manifolds into Riemannian manifolds.

Let $\left(M, \theta, \xi, J, g_{\theta}\right)$ be a strictly pseudoconvex $C R$ manifold of dimension $m=2 d+1 \geq 3$, endowed with its Tanaka-Webster connection $\nabla$ and $\left(N, g^{\prime}\right)$ be a $n$-dimensional Riemannian manifold endowed with a metric connection $\widehat{\nabla}^{\prime}$ on $T N$ with torsion $\widehat{T}^{\prime}$.

Let $\phi: M \rightarrow N$ be a differentiable map and $\phi^{*} T N$ the pull-back bundle endowed with the metric and the connection induced by those of $T N$. The canonical isomorphism $\wedge^{*} M \simeq C l(M)$ allows to consider the bundle $\wedge^{*} M \otimes \phi^{*} T N$ as a Dirac bundle over $M$. Note that $d \phi$ is a section of this Dirac bundle, more precisely, a $\phi^{*} T N$-valued 1-form. The covariant derivative of any $\phi^{*} T N$-valued 1 -form $\sigma$ is given by:

$$
\left(\nabla_{X} \sigma\right)(Y)=\widehat{\nabla}_{X}^{\prime \phi^{*} T N} \sigma(Y)-\sigma\left(\nabla_{X} Y\right)
$$


where $\widehat{\nabla}^{\prime \phi^{*} T N}$ denotes the connection induced by $\widehat{\nabla}^{\prime}$ on $\phi^{*} T N$. The exterior derivative and the divergence of $\sigma$ are respectively denoted by $d^{\widehat{\nabla}^{\prime}} \sigma$ and $\delta^{\hat{\nabla}^{\prime}} \sigma$. The expressions are given by formulas (9) and (10).

Lemma 3.1. For any map $\phi: M \rightarrow N$ :

$$
\begin{aligned}
\mathcal{D} d \phi= & \delta^{\widehat{\nabla}^{\prime}}(d \phi)_{H}-\left(\nabla_{\xi} d \phi\right)(\xi)-\left(\phi^{*} \widehat{T}^{\prime}\right)_{H}-\omega_{\theta} \otimes d \phi(\xi) \\
& +\theta \wedge\left(d \phi \circ \tau-i(\xi)\left(\phi^{*} \widehat{T}^{\prime}\right)\right)
\end{aligned}
$$

and

$$
\begin{aligned}
\mathcal{D}_{J}(d \phi)= & -\Lambda_{\theta}\left(\left(\phi^{*} \widehat{T}^{\prime}\right)_{H}+\omega_{\theta} \otimes d \phi(\xi)\right)+d_{H}^{\widehat{\nabla}^{\prime}} J d \phi-\left(\phi^{*} \widehat{T}^{\prime}\right)_{H}^{J} \\
& +\theta \wedge J\left(\nabla_{\xi} d \phi+i(\xi)\left(\phi^{*} \widehat{T}^{\prime}\right)-d \phi \circ \tau\right)
\end{aligned}
$$

where

$d_{H}^{\widehat{\nabla}^{\prime}} J d \phi=\left(d^{\widehat{\nabla}^{\prime}} J d \phi\right)_{H},\left(\phi^{*} \widehat{T}^{\prime}\right)_{H}^{J}(X, Y)=\left(\phi^{*} \widehat{T}^{\prime}\right)_{H}(J X, Y)+\left(\phi^{*} \widehat{T}^{\prime}\right)_{H}(X, J Y)$

and $\Lambda_{\theta}(\varphi)=\frac{1}{2} \operatorname{trace}_{g_{\theta}} \varphi(., J$.$) .$

Proof. The Dirac operator $\mathcal{D}$ associated to the Dirac bundle $\wedge^{*} M \otimes \phi^{*} T N$ coincides with the operator $\mathcal{A}(\nabla)+\delta^{\hat{\nabla}^{\prime}}$, where $\mathcal{A}(\nabla)$ is the anti-symmetrisation of the covariant derivative on $\wedge^{*} M \otimes \phi^{*} T N$. Hence, $\mathcal{D} d \phi=\delta^{\widehat{\nabla}^{\prime}} d \phi+$ $\mathcal{A}(\nabla) d \phi$. First, we have

$$
\begin{aligned}
\left(\nabla_{X} d \phi\right)(Y) & =\left(\nabla_{X} d \phi\right)_{H}(Y)+\theta(Y)\left(\nabla_{X} d \phi\right)(\xi) \\
& =\left(\nabla_{X}(d \phi)_{H}\right)(Y)+\theta(Y)\left(\nabla_{X} d \phi\right)(\xi) .
\end{aligned}
$$

Hence,

$$
\begin{aligned}
\delta^{\hat{\nabla}^{\prime}} d \phi & =- \text { trace }_{g_{\theta}}(\nabla . d \phi)(.)=- \text { trace }_{g_{\theta}}\left(\nabla .(d \phi)_{H}\right)(.)-\left(\nabla_{\xi} d \phi\right)(\xi) \\
& =\delta^{\hat{\nabla}^{\prime}}(d \phi)_{H}-\left(\nabla_{\xi} d \phi\right)(\xi) .
\end{aligned}
$$

Now, for any $X, Y \in T M$, we have:

$$
(\mathcal{A}(\nabla) d \phi)(X, Y)=\left(\nabla_{X} d \phi\right)(Y)-\left(\nabla_{Y} d \phi\right)(X) .
$$

Formula (9) yields:

$$
\left(d^{\hat{\nabla}^{\prime}} d \phi\right)(X, Y)=(\mathcal{A}(\nabla) d \phi)(X, Y)-d \phi(T(X, Y)) .
$$


Now, we have

$$
\widehat{\nabla}_{X}^{\prime \phi^{*} T N} d \phi(Y)-\widehat{\nabla}_{Y}^{\phi^{*} T N} d \phi(X)-d \phi([X, Y])=-\widehat{T}^{\prime}(d \phi(X), d \phi(Y)) .
$$

Hence

$\left(d^{\widehat{\nabla}^{\prime}} d \phi\right)(X, Y)=\widehat{\nabla}_{X}^{\phi^{*} T N} d \phi(Y)-\widehat{\nabla}_{Y}^{\prime \phi^{*} T N} d \phi(X)-d \phi([X, Y])=-\phi^{*} \widehat{T}^{\prime}(X, Y)$.

Since $T=-\omega_{\theta} \otimes \xi+\theta \wedge \tau$, we deduce that

$$
\begin{aligned}
(\mathcal{A}(\nabla) d \phi)(X, Y)= & -\phi^{*} \widehat{T}^{\prime}(X, Y)-\omega_{\theta}(X, Y) d \phi(\xi)+(\theta \wedge d \phi \circ \tau)(X, Y) \\
= & -\left(\phi^{*} \widehat{T}^{\prime}\right)_{H}(X, Y)-\omega_{\theta}(X, Y) d \phi(\xi) \\
& +\left(\theta \wedge\left(d \phi \circ \tau-i(\xi)\left(\phi^{*} \widehat{T}^{\prime}\right)\right)\right)(X, Y) .
\end{aligned}
$$

The first formula holds. Now, $\mathcal{D}_{J} d \phi$ coincides with $\mathcal{A}(\nabla \circ J) d \phi+\delta^{\hat{\nabla}^{\prime}} J d \phi$, where

$$
(\mathcal{A}(\nabla \circ J) d \phi)(X, Y)=\left(\nabla_{J X} d \phi\right)(Y)-\left(\nabla_{J Y} d \phi\right)(X)
$$

and

$$
\delta^{\widehat{\nabla}^{\prime}} J d \phi=- \text { trace }_{g_{\theta}}(\nabla . J d \phi)(.)=- \text { trace }_{g_{\theta}}\left(\nabla_{J} . d \phi\right)(.) .
$$

Using (13) and the fact that $J$ is parallel, we obtain

$$
\begin{aligned}
( & \mathcal{A}(\nabla \circ J) d \phi)(X, Y) \\
= & \left(\nabla_{Y} d \phi\right)(J X)-\left(\nabla_{X} d \phi\right)(J Y)-\theta(Y)\left(d \phi \circ \tau-i(\xi)\left(\phi^{*} \widehat{T}^{\prime}\right)\right)(J X) \\
& +\theta(X)\left(d \phi \circ \tau-i(\xi)\left(\phi^{*} \widehat{T}^{\prime}\right)\right)(J Y)-\left(\phi^{*} \widehat{T}^{\prime}\right)_{H}(X, J Y)+\left(\phi^{*} \widehat{T}^{\prime}\right)_{H}(Y, J X) \\
= & \left(\nabla_{X} J d \phi\right)(Y)-\left(\nabla_{Y} J d \phi\right)(X)-\left(\phi^{*} \widehat{T}^{\prime}\right)_{H}^{J}(X, Y) \\
& -\left(\theta \wedge J\left(d \phi \circ \tau-i(\xi)\left(\phi^{*} \widehat{T}^{\prime}\right)\right)\right)(X, Y) \\
= & (\mathcal{A}(\nabla) J d \phi)(X, Y)-\left(\phi^{*} \widehat{T}^{\prime}\right)_{H}^{J}(X, Y) \\
& -\left(\theta \wedge J\left(d \phi \circ \tau-i(\xi)\left(\phi^{*} \widehat{T}^{\prime}\right)\right)\right)(X, Y) \\
= & (\mathcal{A}(\nabla) J d \phi)_{H}(X, Y)+\left(\theta \wedge \nabla_{\xi} J d \phi\right)(X, Y) \\
& -\left(\phi^{*} \widehat{T}^{\prime}\right)_{H}^{J}(X, Y)-\left(\theta \wedge J\left(d \phi \circ \tau-i(\xi)\left(\phi^{*} \widehat{T}^{\prime}\right)\right)\right)(X, Y) \\
= & (\mathcal{A}(\nabla) J d \phi)_{H}(X, Y)-\left(\phi^{*} \widehat{T}^{\prime}\right)_{H}^{J}(X, Y) \\
& +\left(\theta \wedge J\left(\nabla_{\xi} d \phi+i(\xi)\left(\phi^{*} \widehat{T}^{\prime}\right)-d \phi \circ \tau\right)\right)(X, Y) .
\end{aligned}
$$

Formula (9) yields:

$$
\left(d \hat{\nabla}_{H}^{\prime} J d \phi\right)(X, Y)=\left(d^{\widehat{\nabla}^{\prime}} J d \phi\right)_{H}(X, Y)=(\mathcal{A}(\nabla) J d \phi)_{H}(X, Y) .
$$


We deduce that

$$
\mathcal{A}(\nabla \circ J) d \phi=d_{H}^{\widehat{\nabla}^{\prime}} J d \phi-\left(\phi^{*} \widehat{T}^{\prime}\right)_{H}^{J}+\theta \wedge J\left(\nabla_{\xi} d \phi+i(\xi)\left(\phi^{*} \widehat{T}^{\prime}\right)-d \phi \circ \tau\right) .
$$

Now, with respect to an adapted frame $\left\{\epsilon_{1}, \ldots \epsilon_{d}, J \epsilon_{1}, \ldots J \epsilon_{d}, \xi\right\}$ :

$$
\delta^{\widehat{\nabla}^{\prime}} J d \phi=-\sum_{i=1}^{d}\left(\left(\nabla_{J \epsilon_{i}} d \phi\right)\left(\epsilon_{i}\right)-\left(\nabla_{\epsilon_{i}} d \phi\right)\left(J \epsilon_{i}\right)\right) .
$$

Using (13), we obtain:

$$
\begin{aligned}
\delta^{\widehat{\nabla}^{\prime} J d \phi=} & -\sum_{i=1}^{d}\left(\left(\nabla_{\epsilon_{i}} d \phi\right)\left(J \epsilon_{i}\right)-\left(\nabla_{J \epsilon_{i}} d \phi\right)\left(\epsilon_{i}\right)\right) \\
& +\sum_{i=1}^{d}\left(\left(\phi^{*} \widehat{T}^{\prime}\right)_{H}\left(J \epsilon_{i}, \epsilon_{i}\right)+\omega_{\theta}\left(J \epsilon_{i}, \epsilon_{i}\right) d \phi(\xi)\right) \\
& -\sum_{i=1}^{d}\left(\left(\phi^{*} \widehat{T}^{\prime}\right)_{H}\left(\epsilon_{i}, J \epsilon_{i}\right)+\omega_{\theta}\left(\epsilon_{i}, J \epsilon_{i}\right) d \phi(\xi)\right) \\
= & -\delta^{\widehat{\nabla}^{\prime}} J d \phi-2 \Lambda_{\theta}\left(\left(\phi^{*} \widehat{T}^{\prime}\right)_{H}+\omega_{\theta} \otimes d \phi(\xi)\right) .
\end{aligned}
$$

Hence the second formula.

From now, we assume that the curvature of the metric connection on $N$ satisfies the first Bianchi identity. This allows to consider the notions of curvature tensor and curvature operator. In the following, we denote respectively by $\widehat{R}^{\prime}$ and $\widehat{\rho}$ the curvature tensor and the curvature operator of $N$. The natural extensions of $\hat{\rho}$ and $\langle$,$\rangle to \wedge^{*} T_{y}^{\mathbb{C}} N$ (where $T_{y}^{\mathbb{C}} N$ is the complexification of $T_{y} N$ ) will be respectively denoted by $\widehat{\rho}_{\mathbb{C}}$ and $($,$) .$

Proposition 3.1 (Corlette type formula). Let $M$ be a compact strictly pseudoconvex $C R$ manifold. For any map $\phi: M \rightarrow N$ such that $i(\xi)\left(\phi^{*} \widehat{T}^{\prime}\right)=$ 0 , we have:

$$
\begin{aligned}
& \int_{M}\left|\nabla_{\xi}(d \phi)_{H}\right|^{2}+\frac{1}{2}\left|\left(\nabla_{\xi} d \phi\right)(\xi)\right|^{2}+\frac{1}{2}\left|\delta^{\hat{\nabla}^{\prime}}(d \phi)_{H}\right|^{2}-\frac{1}{2}\left|\delta^{\hat{\nabla}^{\prime}} d \phi\right|^{2}-|d \phi \circ \tau|^{2} v_{g_{\theta}} \\
& =\int_{M}\left(\phi^{*} \widehat{R}^{\prime}\right)_{\xi} v_{g_{\theta}}
\end{aligned}
$$

where

$$
\left(\phi^{*} \widehat{R}^{\prime}\right)_{\xi}=\operatorname{trace}_{g_{\theta}} \phi^{*} \widehat{R}^{\prime}(., \xi, ., \xi)
$$


Proposition 3.2 (Siu-Sampson type formula). Let $M$ be a compact strictly pseudoconvex $C R$ manifold. For any map $\phi: M \rightarrow N$ such that $\left(\phi^{*} \widehat{T}^{\prime}\right)_{H}^{J}=0$, we have:

$$
\int_{M}\left|d_{H}^{\widehat{\nabla}^{\prime}} J d \phi\right|^{2}-\left|\delta^{\widehat{\nabla}^{\prime}}(d \phi)_{H}\right|^{2}+T(\phi) v_{g_{\theta}}=4 \int_{M}\left(\phi^{*} \hat{\rho}_{\mathbb{C}}^{\prime}\right)_{H}^{-} v_{g_{\theta}},
$$

where

$$
\begin{aligned}
T(\phi)= & \left|\Lambda_{\theta}\left(\left(\phi^{*} \widehat{T}^{\prime}\right)_{H}+\omega_{\theta} \otimes d \phi(\xi)\right)\right|^{2}-\left|\left(\phi^{*} \widehat{T}^{\prime}\right)_{H}+\omega_{\theta} \otimes d \phi(\xi)\right|^{2} \\
& +2(d-1)\langle d \phi \circ J \circ \tau, d \phi\rangle,
\end{aligned}
$$

and where, in an adapted frame $\left\{\epsilon_{1}, \ldots \epsilon_{d}, J \epsilon_{1}, \ldots J \epsilon_{d}, \xi\right\}$,

$$
\left(\phi^{*} \widehat{\rho}_{\mathbb{C}}^{\prime}\right)_{H}^{-}=\sum_{i, j \leq d}\left(\widehat{\rho}_{\mathbb{C}}^{\prime}\left(\eta_{i j}^{\phi}\right), \overline{\eta_{i j}^{\phi}}\right)
$$

with $\eta_{i j}^{\phi}=d \phi\left(Z_{i}\right) \wedge d \phi\left(Z_{j}\right), Z_{i}=\frac{1}{\sqrt{2}}\left(\epsilon_{i}+\sqrt{-1} J \epsilon_{i}\right)$.

The proof of these propositions needs some lemmas.

Lemma 3.2. For any map $\phi: M \rightarrow N$ such that $i(\xi)\left(\phi^{*} \widehat{T}^{\prime}\right)=0$, the following formula holds:

$$
\begin{aligned}
\left\langle\mathcal{D}_{\tau} d \phi, \xi . d \phi\right\rangle= & -\delta \gamma_{\phi}-2\left\langle\nabla_{\xi} d \phi, d \phi \circ \tau\right\rangle \\
& +2|d \phi \circ \tau|^{2}+\langle d \phi(\operatorname{Ric}(\xi)), d \phi(\xi)\rangle .
\end{aligned}
$$

where $\gamma_{\phi}$ is the 1-form defined by $\gamma_{\phi}(X)=\langle(d \phi \circ \tau)(X), d \phi(\xi)\rangle$.

Proof. We have:

$$
\left\langle\mathcal{D}_{\tau} d \phi, \xi . d \phi\right\rangle=\sum_{i}\left\langle\tau\left(e_{i}\right) \cdot \nabla_{e_{i}} d \phi, \xi . d \phi\right\rangle .
$$

Now, for all $\alpha, \beta \in \Omega^{1}(M)$ and all $\sigma, \gamma \in \Omega^{1}\left(\phi^{*} T N\right)$, we have (cf. [9]):

$$
\langle\alpha . \sigma, \beta . \gamma\rangle=\langle\alpha, \beta\rangle\langle\sigma, \gamma\rangle+\langle i(\sigma) \alpha, i(\gamma) \beta\rangle-\langle i(\sigma) \beta, i(\gamma) \alpha\rangle .
$$

Hence,

$$
\begin{aligned}
\left\langle\mathcal{D}_{\tau} d \phi, \xi . d \phi\right\rangle= & \sum_{i}\left(\left\langle\tau\left(e_{i}\right), \xi\right\rangle\left\langle\nabla_{e_{i}} d \phi, d \phi\right\rangle+\left\langle\left(\nabla_{e_{i}} d \phi\right)\left(\tau\left(e_{i}\right)\right), d \phi(\xi)\right\rangle\right. \\
& \left.-\left\langle\left(\nabla_{e_{i}} d \phi\right)(\xi), d \phi\left(\tau\left(e_{i}\right)\right)\right\rangle\right) .
\end{aligned}
$$


We have, on the one hand, $\left\langle\tau\left(e_{i}\right), \xi\right\rangle=0$, and, on the other hand, $\left(\nabla_{X} d \phi\right)(\tau(Y))=\left(\nabla_{X} d \phi \circ \tau\right)(Y)-d \phi\left(\left(\nabla_{X} \tau\right)(Y)\right)$.

Hence (18) becomes:

$$
\begin{aligned}
\left\langle\mathcal{D}_{\tau} d \phi, \xi . d \phi\right\rangle= & \sum_{i}\left\langle\left(\nabla_{e_{i}} d \phi \circ \tau\right)\left(e_{i}\right), d \phi(\xi)\right\rangle-\sum_{i}\left\langle\left(\nabla_{e_{i}} d \phi\right)(\xi),(d \phi \circ \tau)\left(e_{i}\right)\right\rangle \\
& +\langle d \phi(\delta \tau), d \phi(\xi)\rangle .
\end{aligned}
$$

Consider the 1-form $\gamma_{\phi}$ defined by $\gamma_{\phi}(X)=\langle(d \phi \circ \tau)(X), d \phi(\xi)\rangle$, then

$$
\begin{aligned}
\delta \gamma_{\phi}= & -\sum_{i}\left(\nabla_{e_{i}} \gamma_{\phi}\right)\left(e_{i}\right)=-\sum_{i}\left(e_{i} \gamma_{\phi}\left(e_{i}\right)-\gamma_{\phi}\left(\nabla_{e_{i}} e_{i}\right)\right) \\
= & -\sum_{i}\left(e_{i}\left\langle(d \phi \circ \tau)\left(e_{i}\right), d \phi(\xi)\right\rangle-\left\langle(d \phi \circ \tau)\left(\nabla_{e_{i}} e_{i}\right), d \phi(\xi)\right\rangle\right) \\
= & -\sum_{i}\left\langle\widehat{\nabla}_{e_{i}}^{\phi^{*} T N}(d \phi \circ \tau)\left(e_{i}\right)-(d \phi \circ \tau)\left(\nabla_{e_{i}} e_{i}\right), d \phi(\xi)\right\rangle \\
& -\sum_{i}\left\langle\left(\nabla_{e_{i}} d \phi\right)(\xi),(d \phi \circ \tau)\left(e_{i}\right)\right\rangle \\
(19)= & -\sum_{i}\left\langle\left(\nabla_{e_{i}} d \phi \circ \tau\right)\left(e_{i}\right), d \phi(\xi)\right\rangle-\sum_{i}\left\langle\left(\nabla_{e_{i}} d \phi\right)(\xi),(d \phi \circ \tau)\left(e_{i}\right)\right\rangle .
\end{aligned}
$$

Using (19) and the equality $\delta \tau=\operatorname{Ric}(\xi)$, we obtain:

$$
\begin{aligned}
& \left\langle\mathcal{D}_{\tau} d \phi, \xi . d \phi\right\rangle \\
& =-\delta \gamma_{\phi}-2 \sum_{i}\left\langle\left(\nabla_{e_{i}} d \phi\right)(\xi),(d \phi \circ \tau)\left(e_{i}\right)\right\rangle+\langle d \phi(\operatorname{Ric}(\xi)), d \phi(\xi)\rangle .
\end{aligned}
$$

Using (13) and the assumption $i(\xi)\left(\phi^{*} \widehat{T}^{\prime}\right)=0$, we have

$$
\begin{aligned}
\sum_{i \leq m}\left\langle\left(\nabla_{e_{i}} d \phi\right)(\xi),(d \phi \circ \tau)\left(e_{i}\right)\right\rangle & =\sum_{i \leq m}\left\langle\left(\nabla_{\xi} d \phi-d \phi \circ \tau\right)\left(e_{i}\right),(d \phi \circ \tau)\left(e_{i}\right)\right\rangle \\
& =\left\langle\nabla_{\xi} d \phi, d \phi \circ \tau\right\rangle-|d \phi \circ \tau|^{2} .
\end{aligned}
$$

By substituting it in (20), we obtain the required expression.

Lemma 3.3. We have

$$
\left\langle\mathcal{R}_{\xi} d \phi, d \phi\right\rangle=-\left(\phi^{*} \widehat{R}^{\prime}\right)_{\xi}+\frac{1}{2}\langle d \phi(\operatorname{Ric}(\xi)), d \phi(\xi)\rangle
$$

and

$$
\left\langle\mathcal{R}_{H}^{-} d \phi, d \phi\right\rangle=-2\left(\phi^{*} \widehat{\rho}_{\mathbb{C}}^{\prime}\right)_{H}^{-}+(d-1)\langle d \phi \circ J \circ \tau, d \phi\rangle
$$


Proof. We have:

$$
\left\langle\mathcal{R}_{\xi} d \phi, d \phi\right\rangle=-\frac{1}{2} \sum_{i}\left\langle\xi . e_{i} \cdot R\left(\xi, e_{i}\right) d \phi, d \phi\right\rangle=\frac{1}{2} \sum_{i}\left\langle e_{i} \cdot R\left(\xi, e_{i}\right) d \phi, \xi . d \phi\right\rangle .
$$

Using (17), we obtain

$$
\left\langle\mathcal{R}_{\xi} d \phi, d \phi\right\rangle=\frac{1}{2} \sum_{i}\left(\left\langle\left(R\left(\xi, e_{i}\right) d \phi\right)\left(e_{i}\right), d \phi(\xi)\right\rangle-\left\langle\left(R\left(\xi, e_{i}\right) d \phi\right)(\xi), d \phi\left(e_{i}\right)\right\rangle\right) .
$$

Now, we have

$$
\begin{aligned}
(R(X, Y) d \phi)(Z) & =\widehat{R}^{\phi^{*} T N}(X, Y) d \phi(Z)-d \phi(R(X, Y) Z) \\
& =\widehat{R}^{\prime}(d \phi(X), d \phi(Y)) d \phi(Z)-d \phi(R(X, Y) Z) .
\end{aligned}
$$

We deduce from (23) that

$$
\begin{aligned}
\left\langle\mathcal{R}_{\xi} d \phi, d \phi\right\rangle= & \frac{1}{2} \sum_{i}\left(-2 \phi^{*} \widehat{R}^{\prime}\left(\xi, e_{i}, \xi, e_{i}\right)-\left\langle d \phi\left(R\left(\xi, e_{i}\right) e_{i}\right), d \phi(\xi)\right\rangle\right. \\
& \left.+\left\langle d \phi\left(R\left(\xi, e_{i}\right) \xi\right), d \phi\left(e_{i}\right)\right\rangle\right) \\
= & -\sum_{i} \phi^{*} \widehat{R}^{\prime}\left(\xi, e_{i}, \xi, e_{i}\right)+\frac{1}{2}\langle d \phi(\operatorname{Ric}(\xi)), d \phi(\xi)\rangle .
\end{aligned}
$$

Hence the first formula. Now, always using (17) we have:

$$
\begin{aligned}
& \left\langle\mathcal{R}_{H}^{-} d \phi, d \phi\right\rangle \\
& =-\frac{1}{2} \sum_{i, j}\left\langle e_{i} \cdot e_{j} \cdot R_{H}^{-}\left(e_{i}, e_{j}\right) d \phi, d \phi\right\rangle \\
& =-\sum_{i, j}\left\langle\left(R_{H}^{-}\left(e_{i}, e_{j}\right)(d \phi)_{H}\right)\left(e_{i}\right),(d \phi)_{H}\left(e_{j}\right)\right\rangle \\
& =-\frac{1}{2} \sum_{i, j}\left\langle\left(R_{H}\left(e_{i}, e_{j}\right)(d \phi)_{H}-R_{H}\left(J e_{i}, J e_{j}\right)(d \phi)_{H}\right)\left(e_{i}\right),(d \phi)_{H}\left(e_{j}\right)\right\rangle .
\end{aligned}
$$

Using (23) together with the Bianchi identity for $\widehat{R}^{\prime}$, the previous expression becomes (cf. [9]):

$$
\begin{aligned}
\left\langle\mathcal{R}_{H}^{-} d \phi, d \phi\right\rangle & \\
= & -\frac{1}{2} \sum_{i, j}\left(\left(\phi^{*} \widehat{R}^{\prime}\right)_{H}\left(e_{i}, e_{j}, e_{i}, e_{j}\right)-\frac{1}{2}\left(\phi^{*} \widehat{R}^{\prime}\right)_{H}\left(e_{i}, J e_{i}, e_{j}, J e_{j}\right)\right) \\
& +\frac{1}{2} \sum_{i, j}\left\langle(d \phi)_{H}\left(R_{H}\left(e_{i}, e_{j}\right) e_{i}-R_{H}\left(J e_{i}, J e_{j}\right) e_{i}\right),(d \phi)_{H}\left(e_{j}\right)\right\rangle,
\end{aligned}
$$


where $\left(\phi^{*} \widehat{R}^{\prime}\right)_{H}=\left(\phi^{*} \widehat{R}^{\prime}\right) \circ \Pi$. Now, in an adapted frame $\left\{\epsilon_{1}, \ldots, \epsilon_{d}\right.$, $\left.J \epsilon_{1}, \ldots, J \epsilon_{d}, \xi\right\}$ :

$$
\begin{aligned}
& \left\langle\mathcal{R}_{H}^{-} d \phi, d \phi\right\rangle \\
& =-\frac{1}{2} \sum_{i, j \leq d}\left(\phi^{*} \widehat{R}^{\prime}\left(\epsilon_{i}, \epsilon_{j}, \epsilon_{i}, \epsilon_{j}\right)\right. \\
& \quad+\phi^{*} \widehat{R}^{\prime}\left(J \epsilon_{i}, \epsilon_{j}, J \epsilon_{i}, \epsilon_{j}\right)+\phi^{*} \widehat{R}^{\prime}\left(\epsilon_{i}, J \epsilon_{j}, \epsilon_{i}, J \epsilon_{j}\right) \\
& \left.\quad+\phi^{*} \widehat{R}^{\prime}\left(J \epsilon_{i}, J \epsilon_{j}, J \epsilon_{i}, J \epsilon_{j}\right)-2 \phi^{*} \widehat{R}^{\prime}\left(\epsilon_{i}, J \epsilon_{i}, \epsilon_{j}, J \epsilon_{j}\right)\right) \\
& +\frac{1}{2} \sum_{i \leq d}\left(\left\langle d \phi\left(\operatorname{Ric}\left(\epsilon_{i}\right)+J \operatorname{Ric}\left(J \epsilon_{i}\right)\right), d \phi\left(\epsilon_{i}\right)\right\rangle\right. \\
& \left.\quad+\left\langle d \phi\left(\operatorname{Ric}\left(J \epsilon_{i}\right)-J \operatorname{Ric}\left(\epsilon_{i}\right)\right), d \phi\left(J \epsilon_{i}\right)\right\rangle\right) .
\end{aligned}
$$

The first five curvature terms of $(24)$ are $-2 \sum_{i, j \leq d}\left(\hat{\rho}_{\mathbb{C}}^{\prime}\left(\eta_{i j}^{\phi}\right), \overline{\eta_{i j}^{\phi}}\right)$. Finally, using (1), we obtain:

$$
\begin{aligned}
& \frac{1}{2} \sum_{i \leq d}\left(\left\langle d \phi\left(\operatorname{Ric}\left(\epsilon_{i}\right)+J \operatorname{Ric}\left(J \epsilon_{i}\right)\right), d \phi\left(\epsilon_{i}\right)\right\rangle\right. \\
& \left.\quad+\left\langle d \phi\left(\operatorname{Ric}\left(J \epsilon_{i}\right)-J \operatorname{Ric}\left(\epsilon_{i}\right)\right), d \phi\left(J \epsilon_{i}\right)\right\rangle\right)=(d-1)\langle d \phi \circ J \circ \tau, d \phi\rangle,
\end{aligned}
$$

this concludes the proof.

Proof of Proposition 3.1. Using (7), we have:

$$
\frac{1}{2} \int_{M}\left\langle\left[\mathcal{D}^{2}, \lambda_{\xi}\right] d \phi, \xi . d \phi\right\rangle v_{g_{\theta}}=\int_{M}\left\langle\mathcal{D}_{\tau} d \phi, \xi . d \phi\right\rangle-2\left\langle\mathcal{R}_{\xi} d \phi, d \phi\right\rangle v_{g_{\theta}} .
$$

Now, using (2) we have

$$
\begin{aligned}
\int_{M}\left\langle\left[\mathcal{D}^{2}, \lambda_{\xi}\right] d \phi, \xi . d \phi\right\rangle v_{g_{\theta}} & =\int_{M}\left\langle\mathcal{D}^{2}(\xi . d \phi), \xi . d \phi\right\rangle-\left\langle\mathcal{D}^{2} d \phi, d \phi\right\rangle v_{g_{\theta}} \\
& =\int_{M}|\mathcal{D}(\xi . d \phi)|^{2}-|\mathcal{D}(d \phi)|^{2} v_{g_{\theta}} \\
& =\int_{M}\left|\xi \cdot \mathcal{D}(d \phi)+2 \nabla_{\xi} d \phi\right|^{2}-|\mathcal{D}(d \phi)|^{2} v_{g_{\theta}} \\
& =4 \int_{M}\left\langle\xi \cdot \mathcal{D}(d \phi), \nabla_{\xi} d \phi\right\rangle+\left|\nabla_{\xi} d \phi\right|^{2} v_{g_{\theta}} .
\end{aligned}
$$


Equation (11) gives:

$$
\begin{aligned}
\left\langle\xi \cdot \mathcal{D}(d \phi), \nabla_{\xi} d \phi\right\rangle= & \left\langle\left(\nabla_{\xi} d \phi\right)(\xi), \delta^{\widehat{\nabla}^{\prime}}(d \phi)_{H}\right\rangle \\
& -\left|\left(\nabla_{\xi} d \phi\right)(\xi)\right|^{2}-\left\langle\nabla_{\xi} d \phi, d \phi \circ \tau-i(\xi)\left(\phi^{*} \widehat{T}^{\prime}\right)\right\rangle .
\end{aligned}
$$

Since

$$
\begin{aligned}
\left|\delta^{\widehat{\nabla}^{\prime}} d \phi\right|^{2} & =\left|\delta^{\widehat{\nabla}^{\prime}}(d \phi)_{H}-\left(\nabla_{\xi} d \phi\right)(\xi)\right|^{2} \\
& =\left|\delta^{\widehat{\nabla}^{\prime}}(d \phi)_{H}\right|^{2}+\left|\left(\nabla_{\xi} d \phi\right)(\xi)\right|^{2}-2\left\langle\left(\nabla_{\xi} d \phi\right)(\xi), \delta^{\widehat{\nabla}^{\prime}}(d \phi)_{H}\right\rangle
\end{aligned}
$$

and $i(\xi)\left(\phi^{*} \widehat{T}^{\prime}\right)=0$, we deduce that

$$
\begin{aligned}
\left\langle\xi \cdot \mathcal{D}(d \phi), \nabla_{\xi} d \phi\right\rangle= & \frac{1}{2}\left|\delta^{\hat{\nabla}^{\prime}}(d \phi)_{H}\right|^{2}-\frac{1}{2}\left|\delta^{\hat{\nabla}^{\prime}} d \phi\right|^{2} \\
& -\frac{1}{2}\left|\left(\nabla_{\xi} d \phi\right)(\xi)\right|^{2}-\left\langle\nabla_{\xi} d \phi, d \phi \circ \tau\right\rangle .
\end{aligned}
$$

Hence,

$$
\begin{aligned}
\int_{M}\left\langle\left[\mathcal{D}^{2}, \lambda_{\xi}\right] d \phi, \xi \cdot d \phi\right\rangle v_{g_{\theta}}= & 4 \int_{M}\left|\nabla_{\xi}(d \phi)_{H}\right|^{2}+\frac{1}{2}\left|\delta^{\nabla^{\prime}}(d \phi)_{H}\right|^{2}-\frac{1}{2}\left|\delta^{\widehat{\nabla}^{\prime}} d \phi\right|^{2} \\
(26) & +\frac{1}{2}\left|\left(\nabla_{\xi} d \phi\right)(\xi)\right|^{2}-\left\langle\nabla_{\xi} d \phi, d \phi \circ \tau\right\rangle v_{g_{\theta}} .
\end{aligned}
$$

Using (16),(21) and (26) in (25), one gets the result.

Proof of Proposition 3.2. Using (8), we have

$$
\frac{1}{2} \int_{M}\left\langle\left\{\mathcal{D}^{2}, \lambda_{\xi}\right\} d \phi, \xi . d \phi\right\rangle v_{g_{\theta}}=\int_{M}\left|\mathcal{D}_{J}(d \phi)\right|^{2}-\left\langle\nabla_{\xi, \xi}^{2} d \phi, d \phi\right\rangle+2\left\langle\mathcal{R}_{H}^{-} d \phi, d \phi\right\rangle v_{g_{\theta}} .
$$

Since for any function $f, \int_{M} \xi f v_{g_{\theta}}=0$,

$$
\int_{M}\left\langle\nabla_{\xi, \xi}^{2} d \phi, d \phi\right\rangle v_{g_{\theta}}=\int_{M}\left(\xi\left\langle\nabla_{\xi} d \phi, d \phi\right\rangle-\left|\nabla_{\xi} d \phi\right|^{2}\right) v_{g_{\theta}}=-\int_{M}\left|\nabla_{\xi} d \phi\right|^{2} v_{g_{\theta}} .
$$

Hence, we have

$$
\int_{M}\left|\mathcal{D}_{J}(d \phi)\right|^{2}+\left|\nabla_{\xi} d \phi\right|^{2}-\frac{1}{2}\left\langle\left\{\mathcal{D}^{2}, \lambda_{\xi}\right\} d \phi, \xi . d \phi\right\rangle v_{g_{\theta}}=-2 \int_{M}\left\langle\mathcal{R}_{H}^{-} d \phi, d \phi\right\rangle v_{g_{\theta}} .
$$


Using (11), we have

$$
\begin{aligned}
& \int_{M}\left\langle\left\{\mathcal{D}^{2}, \lambda_{\xi}\right\} d \phi, \xi . d \phi\right\rangle v_{g_{\theta}} \\
& =\int_{M}|\mathcal{D}(\xi . d \phi)|^{2}+|\mathcal{D}(d \phi)|^{2} v_{g_{\theta}} \\
& =\int_{M} 2|\mathcal{D}(d \phi)|^{2}+4\left\langle\xi \cdot \mathcal{D}(d \phi), \nabla_{\xi} d \phi\right\rangle+4\left|\nabla_{\xi} d \phi\right|^{2} v_{g_{\theta}} \\
& =2 \int_{M}\left|\delta^{\nabla^{\prime}}(d \phi)_{H}\right|^{2}+\left|\nabla_{\xi} d \phi\right|^{2}-\left|\left(\nabla_{\xi} d \phi\right)(\xi)\right|^{2} \\
& \quad+\left|\nabla_{\xi} d \phi-d \phi \circ \tau+i(\xi)\left(\phi^{*} \widehat{T}^{\prime}\right)\right|^{2} \\
& \quad+\left|\left(\phi^{*} \widehat{T}^{\prime}\right)_{H}+\omega_{\theta} \otimes d \phi(\xi)\right|^{2} v_{g_{\theta}} .
\end{aligned}
$$

Using (28) and (12) with $\left(\phi^{*} \widehat{T}^{\prime}\right)_{H}^{J}=0$, the left-hand side of (27) becomes:

$$
\begin{aligned}
& \int_{M}\left|d_{H}^{\widehat{\nabla}^{\prime}} J d \phi\right|^{2}+\left|J\left(\nabla_{\xi} d \phi-d \phi \circ \tau+i(\xi)\left(\phi^{*} \widehat{T}^{\prime}\right)\right)\right|^{2} \\
& \quad+\left|\left(\nabla_{\xi} d \phi\right)(\xi)\right|^{2}-\left|\nabla_{\xi} d \phi-d \phi \circ \tau+i(\xi)\left(\phi^{*} \widehat{T}^{\prime}\right)\right|^{2} \\
& \quad-\left|\delta^{\widehat{\nabla}^{\prime}}(d \phi)_{H}\right|^{2}+\left|\Lambda_{\theta}\left(\left(\phi^{*} \widehat{T}^{\prime}\right)_{H}+\omega_{\theta} \otimes d \phi(\xi)\right)\right|^{2} \\
& \quad-\left|\left(\phi^{*} \widehat{T}^{\prime}\right)_{H}+\omega_{\theta} \otimes d \phi(\xi)\right|^{2} v_{g_{\theta}} .
\end{aligned}
$$

Noting that for any 1-form $\alpha,|\alpha|^{2}=|J \alpha|^{2}+|\alpha(\xi)|^{2}$, we obtain the required expression. Using (22), we obtain the right-hand side of (27) and consequently the formula.

Remark 3.1. Note that if $\widehat{\nabla}^{\prime}$ is the Levi-Civita connection, then $\widehat{T}^{\prime}=0$, and therefore Formulas (14),(15) are valid for any map $\phi: M \rightarrow N$.

\section{Harmonic maps and the geometry of Sasakian manifolds.}

Let $\phi:(M, g) \rightarrow\left(N, g^{\prime}\right)$ be a differential map between Riemannian manifolds. Then, the Hessian of $\phi$ is defined by:

$$
(D d \phi)(X, Y)=\left(D_{X} d \phi\right)(Y)=D_{X}^{\prime \phi^{*} T N} d \phi(Y)-d \phi\left(D_{X} Y\right),
$$

where $D^{\prime}$ (resp. $D$ ) is the Levi-Civita connection on $T N$ (resp. TM). The map $\phi$ is called harmonic if $\Gamma(\phi)=$ trace $_{g}(D d \phi)(.,)=$.0 . 
Lemma 4.1. Let $\left(M, g_{\theta}, \nabla\right)$ be a strictly pseudoconvex $C R$ manifold and $\left(N, g^{\prime}, \widehat{\nabla}^{\prime}\right)$ be a $n$-dimensional Riemannian manifold endowed with a metric connection. Then, for any map $\phi: M \rightarrow N$ we have:

$$
\delta^{\widehat{\nabla}^{\prime}} d \phi=-\Gamma(\phi)-\text { trace }_{g_{\theta}} \phi^{*} \widehat{U}^{\prime},
$$

where $\widehat{U}^{\prime}$ is the 2-tensor $\widehat{U}^{\prime}=\widehat{\nabla}^{\prime}-D^{\prime}$.

Proof. The difference between the Levi-Civita connection $D$ and the TanakaWebster connection $\nabla$ is given by (cf. [6]):

$$
D-\nabla=\frac{1}{2} \theta \odot J+\left(A_{\theta}-\frac{1}{2} \omega_{\theta}\right) \otimes \xi-\tau \otimes \theta,
$$

where $A_{\theta}(X, Y)=g_{\theta}(\tau(X), Y)$ and where $\odot$ denotes the symmetric product (i.e. for any $X, Y \in T M,(\theta \odot J)(X, Y)=\theta(X) J Y+\theta(Y) J X)$. Hence,

$$
\begin{aligned}
\left(\nabla_{X} d \phi\right)(Y)= & \left(D_{X} d \phi\right)(Y)+\phi^{*} \widehat{U}^{\prime}+\frac{1}{2}(\theta \odot d \phi \circ J)(X, Y) \\
& +\left(A_{\theta}(X, Y)-\frac{1}{2} \omega_{\theta}(X, Y)\right) d \phi(\xi)-\theta(Y)(d \phi \circ \tau)(X)
\end{aligned}
$$

Now, taking the trace with respect to an orthonormal tangent frame, we obtain the result.

In the following, we assume that $N$ is a Riemannian manifold endowed with its Levi-Civita connection, hence $\widehat{\nabla}^{\prime}=D^{\prime}, \widehat{U}^{\prime}=0$ and $\widehat{T}^{\prime}=0$. In this case, a harmonic map $\phi: M \rightarrow N$ satisfies $\delta^{\widehat{\nabla}^{\prime}} d \phi=0$.

Theorem 4.1. Let $M$ be a compact Sasakian manifold of dimension $m \geq 3$ and $N$ be a Riemannian manifold with nonpositive sectional curvature. Then any harmonic map $\phi: M \rightarrow N$ satisfies $d \phi(\xi)=0$.

Proof. Let $\phi: M \rightarrow N$ be a harmonic map. Then, under the assumptions $\widehat{T}^{\prime}=0$ and $\tau=0$, we deduce from (11) that $\mathcal{D} d \phi=-\omega_{\theta} \otimes d \phi(\xi)$. Moreover, Equation (14) gives:

$$
\int_{M}\left|\nabla_{\xi}(d \phi)_{H}\right|^{2}+\left|\left(\nabla_{\xi} d \phi\right)(\xi)\right|^{2} v_{g_{\theta}}=\int_{M}\left(\phi^{*} \widehat{R}^{\prime}\right)_{\xi} v_{g_{\theta}} .
$$


Since the sectional curvature of $N$ is nonpositive, $\left(\phi^{*} \widehat{R}^{\prime}\right)_{\xi}$ is nonpositive. Hence, we deduce from the previous equation that $\nabla_{\xi} d \phi=0$. It follows from (13) that

$$
\widehat{\nabla}_{X}^{\phi^{*} T N} d \phi(\xi)=\left(\nabla_{X} d \phi\right)(\xi)=\left(\nabla_{\xi} d \phi\right)(X)=0
$$

Since $\omega_{\theta}$ is parallel, we deduce using (30) that $\mathcal{D}^{2} d \phi=0$. Now, integrating, we obtain

$$
0=\int_{M}\left\langle\mathcal{D}^{2} d \phi, d \phi\right\rangle v_{g_{\theta}}=\int_{M}|\mathcal{D} d \phi|^{2} v_{g_{\theta}}=d \int_{M}|d \phi(\xi)|^{2} v_{g_{\theta}} .
$$

Therefore $d \phi(\xi)=0$.

Suppose that $M$ is a Sasakian manifold which is the total space of Riemannian submersion with minimal fibers over a Kähler manifold. Then, we have

Theorem 4.2. Let $M$ be a compact Sasakian manifold of dimension $m \geq 3$ and $N$ be a Riemannian manifold with nonpositive sectional curvature. Let $(\tilde{M}, \tilde{J})$ be a Kähler manifold and $\pi: M \rightarrow \tilde{M}$ be a Riemannian submersion with minimal fibers. If $d \pi(\xi)=0$, then, for any harmonic map $\phi: M \rightarrow N$, there exists a unique harmonic map $\tilde{\phi}: \tilde{M} \rightarrow N$ such that $\phi=\tilde{\phi} \circ \pi$.

Proof. For any harmonic map $\phi: M \rightarrow N$, we have by Theorem 4.1, $d \phi(\xi)=$ 0 . Since $d \pi(\xi)=0$, it follows from Proposition 4.2 of [12], that there exists a unique map $\tilde{\phi}: \tilde{M} \rightarrow N$ such that $\phi=\tilde{\phi} \circ \pi$. Now, we have

$$
\left(\nabla_{X} d \phi\right)(Y)=\left(D_{d \pi(X)} d \tilde{\phi}\right)(d \pi(Y))+d \tilde{\phi}\left(\left(\nabla_{X} d \pi\right)(Y)\right),
$$

where

$$
\begin{aligned}
& \left(D_{\tilde{X}} d \tilde{\phi}\right)(\tilde{Y})=\widehat{\nabla}_{\tilde{X}}^{\prime \tilde{\phi}^{*} T N} d \tilde{\phi}(\tilde{Y})-d \tilde{\phi}\left(\tilde{D}_{\tilde{X}} \tilde{Y}\right) \quad \text { and } \\
& \left(\nabla_{X} d \pi\right)(Y)=\tilde{D}_{X}^{\pi^{*} T \tilde{M}} d \pi(Y)-d \pi\left(\nabla_{X} Y\right)
\end{aligned}
$$

Since $\pi$ is a harmonic Riemannian submersion, we deduce the harmonicity of $\tilde{\phi}$ by taking the trace in the above formula. 


\section{Application to geometry of Sasakian manifolds.}

The sectional curvature of a Sasakian manifold is always positive when restricted to planes containing $\xi$ (cf. [2]), and consequently, the sectional curvature cannot be nonpositive. This fact arises from a more general result:

Theorem 4.1. On a compact manifold of odd dimension $m \geq 3$, both a Sasakian metric and a metric with nonpositive sectional curvature cannot exist.

Proof. Consider the identity map $I:(M, g) \rightarrow(M, h)$ with $g$ a Sasakian metric and $h$ a metric with nonpositive sectional curvature. In its homotopy class, $I$ contains a harmonic representative (cf. Eells-Sampson [8]) with maximal rank at a point, contradicting Theorem 4.1.

As a consequence of this theorem, we recover the fact that the Heisenberg nilmanifold does not admit any flat metric.

Remark 4.1. The previous results deal with Sasakian manifolds. The assumption Sasakian is a technical assumption which allows to obtain a vanishing theorem (Theorem 4.1). For the moment, we don't know if these results can be extended to strictly pseudoconvex $C R$ manifolds with pseudoHermitian torsion non zero even if we do some assumptions on pseudoHermitian torsion.

\section{Harmonic maps and $C R$-holomorphic maps.}

Definition 5.1. Let $\left(N, g^{\prime}\right)$ be a $m$-dimensional Riemannian manifold endowed with its Levi-Civita connection. Remember that the complex sectional curvature of a 2-plane $P=\mathbb{C}\{Z, W\} \subset T_{y}^{\mathbb{C}} M$, is defined by:

$$
\widehat{K}_{\mathbb{C}}^{\prime}(P)=\widehat{K}_{\mathbb{C}}^{\prime}(Z \wedge W)=\frac{\left(\widehat{\rho}_{\mathbb{C}}^{\prime}(Z \wedge W), \overline{Z \wedge W}\right)}{(Z \wedge W, \overline{Z \wedge W})} .
$$

The sign of the complex sectional curvature always determines the sign of the sectional curvature (cf. [9]). The converse is only true in dimension 3. Note that the assumption of nonpositive (resp. nonnegative) complex sectional curvature is satisfied if the curvature operator is nonpositive (resp. 
nonnegative). In particular, locally symmetric spaces of noncompact type (resp. compact type) are examples of Riemannian manifolds with nonpositive (resp. nonnegative) complex sectional curvature. If $\left(N, g^{\prime}\right)$ is a locally symmetric spaces of noncompact type, the curvature operator of the LeviCivita connection is given by $\hat{\rho}(Z \wedge W)=-[Z, W]$.

Definition 5.2. Let $E$ be a complex vector bundle over a strictly pseudoconvex $C R$ manifold $M$. A holomorphic structure on $E$ is a linear map

$$
\bar{\partial}^{E}: \Gamma(E) \rightarrow \Gamma\left(\left(T^{0,1} M\right)^{*} \otimes E\right)
$$

such that

$$
\bar{\partial} \bar{Z}(f \sigma)=\bar{Z} f \otimes \sigma+f \bar{\partial} \bar{Z} \sigma
$$

and

$$
\left(\bar{\partial}_{\bar{Z}}^{E} \bar{\partial}_{\bar{W}}^{E}-\bar{\partial}_{\bar{W}}^{E} \bar{\partial}_{\bar{Z}}^{E}-\bar{\partial}_{[\bar{Z}, \bar{W}]}^{E}\right)(\sigma)=0,
$$

for all $Z, W \in T^{1,0} M, f \in C_{\mathbb{C}}^{\infty}(M)$ and $\sigma \in \Gamma(E)$.

Let $\partial \phi$ be the restriction of $d \phi$ to $T^{1,0} M$. Remember that $\partial \phi$ is said to be holomorphic if

$$
\left(\nabla_{\bar{W}}^{(0,1)} \partial \phi\right)(Z):=\widehat{\nabla}_{\bar{W}}^{\prime \phi^{*} T^{\mathbb{C}_{N}}} \partial \phi(Z)-\partial \phi\left(\nabla_{\bar{W}} Z\right)=0 .
$$

Theorem 5.1. Let $M$ be a compact Sasakian manifold of dimension $m \geq 3$ and $N$ be a locally symmetric space of noncompact type. Then any harmonic map $\phi: M \rightarrow N$ induces a holomorphic structure on $\phi^{*} T^{\mathbb{C}} N$ given by $\widehat{\nabla}^{\prime \phi^{*} T_{N}}$. Moreover, $\partial \phi$ is a holomorphic $\phi^{*} T^{\mathbb{C}} N$-valued 1 -form.

Proof. Under the assumptions of the Theorem, we have for any harmonic $\operatorname{map} \phi: M \rightarrow N$ :

$$
\begin{aligned}
& \int_{M}\left|\nabla_{\xi}(d \phi)_{H}\right|^{2}+\frac{1}{2}\left|\left(\nabla_{\xi} d \phi\right)(\xi)\right|^{2}+\frac{1}{2}\left|\delta^{\widehat{\nabla}^{\prime}}(d \phi)_{H}\right|^{2} v_{g_{\theta}}=\int_{M}\left(\phi^{*} \widehat{R}^{\prime}\right)_{\xi} v_{g_{\theta}} \\
& \int_{M}\left|d_{H}^{\hat{\nabla}^{\prime}} J d \phi\right|^{2}-\left|\delta^{\hat{\nabla}^{\prime}}(d \phi)_{H}\right|^{2}+d(d-1)|d \phi(\xi)|^{2} v_{g_{\theta}}=4 \int_{M}\left(\phi^{*} \hat{\rho}_{\mathbb{C}}^{\prime}\right)_{H}^{-} v_{g_{\theta}}
\end{aligned}
$$

with

$$
\left(\phi^{*} \widehat{R}^{\prime}\right)_{\xi}=-\sum_{i \leq d}\left|\left[d \phi(\xi), d \phi\left(\overline{Z_{i}}\right)\right]\right|^{2}
$$


and

$$
\left(\phi^{*}{\widehat{\rho_{\mathbb{C}}}}_{\mathrm{C}_{H}}^{-}=-\sum_{i, j \leq d}\left|\left[d \phi\left(\overline{Z_{i}}\right), d \phi\left(\overline{Z_{j}}\right)\right]\right|^{2}\right.
$$

Since $\left(\phi^{*} \widehat{R}^{\prime}\right)_{\xi}$ and $\left(\phi^{*} \widehat{\rho}_{\mathbb{C}}^{\prime}\right)_{H}^{-}$are nonpositive, we deduce by (31) and (32), that $\delta \widehat{\nabla}^{\prime}(d \phi)_{H}=0, d_{H}^{\widehat{\nabla}^{\prime}} J d \phi=0$ and $\left[d \phi\left(\overline{Z_{i}}\right), d \phi\left(\overline{Z_{j}}\right)\right]=0, i, j \leq d$. Hence, for any $Z, W \in T^{1,0} M, \widehat{R}^{\phi^{*} T^{\mathbb{C}_{N}}}(\bar{Z}, \bar{W})=[d \phi(\bar{Z}), d \phi(\bar{W})]=0$. We deduce that $\widehat{\nabla}^{\prime \phi^{*} T^{\mathbb{C}_{N}}}$ defines a holomorphic structure on $\phi^{*} T^{\mathbb{C}} N$. Now, by an easy calculation, we have

$$
\left(\nabla \frac{(0,1)}{W} \partial \phi\right)(Z)=-\left(d_{H}^{\hat{\nabla}^{\prime}} J d \phi\right)(J X, Y)-\sqrt{-1}\left(d_{H}^{\hat{\nabla}^{\prime}} J d \phi\right)(X, Y)=0 .
$$

Hence $\partial \phi$ is a holomorphic $\phi^{*} T^{\mathbb{C}} N$-valued 1-form.

Definition 5.3 (cf. [13]). Let $(M, J)$ be a strictly pseudoconvex $C R$ manifold and $\left(N, J^{\prime}\right)$ be a Kähler manifold. A map $\phi: M \rightarrow N$ is called a $C R$-holomorphic (resp. $C R$-antiholomorphic) map if $d \phi \circ J=J^{\prime} \circ d \phi$ (resp. $\left.d \phi \circ J=-J^{\prime} \circ d \phi\right)$.

Note that a $C R$-holomorphic (resp. $C R$-antiholomorphic) map is always a harmonic map (cf. [13]).

Suppose that $M$ is a Sasakian manifold which is the total space of a $C R$-holomorphic Riemannian submersion over a Kähler manifold. Then, we have

Theorem 5.2. Let $M$ be a compact Sasakian manifold of dimension $m \geq 3$ and $N$ be a locally symmetric space of noncompact type. If $(\tilde{M}, \tilde{J})$ is a Kähler manifold and $\pi: M \rightarrow \tilde{M}$ is a CR-holomorphic Riemannian submersion, then, for any harmonic map $\phi: M \rightarrow N$, there exists a unique harmonic map $\tilde{\phi}: \tilde{M} \rightarrow N$ such that $\phi=\tilde{\phi} \circ \pi$. Moreover, $\tilde{\phi}$ induces a holomorphic structure on $\tilde{\phi}^{*} T^{\mathbb{C}} N$ given by $\widehat{\nabla}^{\prime \tilde{\phi}^{*} T^{\mathbb{C}}}$ and $\partial \tilde{\phi}$ is a holomorphic $\tilde{\phi}^{*} T^{\mathbb{C}} N$ valued 1-form.

As examples of such Sasakian manifolds, we can quote the compact regular Sasakian manifolds. Such a manifold can be realised as the total space of a fibration over a compact Kähler manifold (the Boothby-Wang fibration).

Proof of Theorem 5.2. Since any $C R$-holomorphic Riemannian submersion is a Riemannian submersion with minimal fibers and satisfies $d \pi(\xi)=0$, we deduce from Theorem 4.2 that for any harmonic map 
$\phi: M \rightarrow N$, there exists a unique harmonic map $\tilde{\phi}: \tilde{M} \rightarrow N$ such that $\phi=\tilde{\phi} \circ \pi$. Now, we prove that $d^{\hat{\nabla}^{\prime}} \tilde{J} d \tilde{\phi}=0$ (i.e., $\tilde{\phi}$ is pluriharmonic). Since $\left(\nabla_{X} d \phi\right)(Y)=\left(D_{d \pi(X)} d \tilde{\phi}\right)(d \pi(Y))+d \tilde{\phi}\left(\left(\nabla_{X} d \pi\right)(Y)\right)$ and that $\pi$ is holomorphic, we have:

$$
\left(d_{H}^{\hat{\nabla}^{\prime}} J d \phi\right)(X, Y)=\left(d^{\hat{\nabla}^{\prime}} \tilde{J} d \tilde{\phi}\right)(d \pi(X), d \pi(Y))+d \tilde{\phi}\left(\left(d_{H}^{\tilde{D}} J d \pi\right)(X, Y)\right) .
$$

From $d_{H}^{\widehat{\widehat{\nabla}}^{\prime}} J d \phi=0$, we deduce that

$$
\left(d^{\hat{\nabla}^{\prime}} \tilde{J} d \tilde{\phi}\right)(d \pi(X), d \pi(Y))=-d \tilde{\phi}\left(\left(d_{H}^{\tilde{D}} J d \pi\right)(X, Y)\right) .
$$

Using (13), we obtain

$$
\begin{aligned}
\left(d_{H}^{\tilde{D}} J d \pi\right)(X, Y) & =\left(\nabla_{Y} d \pi\right)(J X)-\left(\nabla_{X} d \pi\right)(J Y) \\
& =\tilde{J}\left(\left(\nabla_{Y} d \pi\right)(X)-\left(\nabla_{X} d \pi\right)(Y)\right) \\
& =-\omega_{\theta}(X, Y) \tilde{J} d \pi(\xi)=0 .
\end{aligned}
$$

Hence, $d^{\widehat{\nabla}^{\prime}} \tilde{J} d \tilde{\phi}=0$ (since $\pi$ is a Riemannian submersion) and $\nabla_{\frac{(0,1)}{W}}^{\partial \tilde{\phi}}=0$.

A rigidity result for harmonic maps from Sasakian manifolds to Kähler manifolds.

Definition 5.4. A Kähler manifold $N$ has a strongly negative curvature (resp. strongly positive curvature) at a point $y \in N$ if the complex sectional curvature $\widehat{K}_{\mathbb{C}}^{\prime}$ at $y$ is negative (resp. positive) on planes $P=\mathbb{C}\{Z, W\} \subset$ $T_{y}^{\mathbb{C}} N$ such that $(Z \wedge W)^{1,1} \neq 0$.

Theorem 5.3. Let $M$ be a compact Sasakian manifold of dimension $m>3$ and $N$ be a Kähler manifold with strongly negative curvature. Then any harmonic map $\phi: M \rightarrow N$ with $\operatorname{rank}_{x}(\phi) \geq 3$ is CR-holomorphic or $C R$ antiholomorphic.

This theorem is an analogue of the Siu theorem (cf. Siu[20]).

Proof of Theorem 5.3. Since $N$ has a strongly negative curvature, the complex sectional curvature is nonpositive. Hence $\left(\phi^{*} \widehat{R}^{\prime}\right)_{\xi}$ and $\left(\phi^{*} \widehat{\rho}_{\mathbb{C}}^{\prime}\right)_{H}^{-}$are nonpositive. As in Theorem 5.1, we obtain that if $\phi$ is harmonic, $d \phi(\xi)=0$ and $\left(\phi^{*} \widehat{\rho}_{\mathbb{C}}\right)_{H}^{-}=0$. Now, the end of the proof follows from the proof of Theorem 4.1 of [9]. 
Corollary 5.1. Let $M$ be a compact regular Sasakian manifold of dimension $m>3$ and $\pi: M \rightarrow \tilde{M}$ be the Boothby-Wang fibration over a compact Kähler manifold $\tilde{M}$. Then any harmonic map with rank $\geq 3$ from $M$ into a Kähler manifold $N$ with strongly negative curvature, factors, via $\pi$, into a unique holomorphic map from $\tilde{M}$ into $N$.

\section{A rigidity result for harmonic maps between Sasakian manifolds.}

In this section, we assume that $\left(M, \theta, \xi, J, g_{\theta}\right)$ and $\left(N, \theta^{\prime}, \xi^{\prime}, J^{\prime}, g_{\theta^{\prime}}^{\prime}\right)$ are Sasakian manifolds endowed with their Tanaka-Webster connections. Hence, $\hat{\nabla}^{\prime}=\nabla^{\prime}$, where $\nabla^{\prime}$ denotes the Tanaka-Webster connection of $N$. Hence $\widehat{U}^{\prime}=-\frac{1}{2}\left(\theta^{\prime} \odot J^{\prime}-\Omega_{\theta^{\prime}} \otimes \xi^{\prime}\right)$ and $\widehat{T}^{\prime}=-\Omega_{\theta^{\prime}} \otimes \xi^{\prime}$.

As we saw it in Paragraphe 4, the complex sectional curvature (for the Levi-Civita connection) of a Sasakian manifold $N$ cannot be nonpositive. Now, since the curvature of the Tanaka-Webster connection of a Sasakian manifold satisfies the Bianchi identity, the complex sectional curvature associated to the Tanaka-Webster connection is well defined. We call it the Tanaka-Webster complex sectional curvature. Note that the parallelism of $J^{\prime}$ and $\xi^{\prime}$ implies that the Tanaka-Webster complex sectional curvature is non-zero only on planes $P=\mathbb{C}\{Z, W\} \subset T_{y}^{\mathbb{C}} N$ such that $(Z \wedge W)^{1,1} \neq 0$. Now, we can define the concept of strongly negative (or positive) curvature for a Sasakian manifold.

Definition 5.5. A Sasakian manifold $N$ has a strongly negative TanakaWebster curvature (resp. strongly positive Tanaka-Webster curvature) at a point $y \in N$ if the Tanaka-Webster complex sectional curvature $\widehat{K}_{\mathbb{C}}^{\prime}$ at $y$ is negative (resp. positive) on planes $P=\mathbb{C}\{Z, W\} \subset T_{y}^{\mathbb{C}} N$ such that $(Z \wedge W)^{1,1} \neq 0$.

Definition 5.6 (cf. [6], [12], [24]). Let $(M, J, H)$ and $\left(N, J^{\prime}, H^{\prime}\right)$ be strictly pseudoconvex $C R$ manifolds. A map $\phi: M \rightarrow N$ is called a $C R$ holomorphic map if $d \phi \circ J=J^{\prime} \circ d \phi$. A map $\phi: M \rightarrow N$ is called a $C R$-map if $d \phi(H) \subset H^{\prime}$ and $(d \phi \circ J)_{H}=\left(J^{\prime} \circ d \phi\right)_{H}$.

Note that a $C R$-holomorphic map is always a $C R$-map, but the converse is, in general, not true. A $C R$-holomorphic map is a harmonic map (cf. [12]). Conversely

Theorem 5.4. Let $M$ be a compact Sasakian manifold of dimension $m>3$ and $N$ be a Sasakian manifold of dimension $n$ with strongly negative Tanaka- 
Webster curvature. Then any harmonic map $\phi: M \rightarrow N$ with $\operatorname{rank}_{x}(\phi) \geq 3$ and such that $\phi^{*} \theta^{\prime}=\theta$, is an isometric and a CR-holomorphic immersion.

Proof. First, since $\phi^{*} \theta^{\prime}=\theta$, we have $\phi^{*} \widehat{U}^{\prime}=-\frac{1}{2}\left(\theta \odot J^{\prime} \circ d \phi-\omega_{\theta} \otimes \xi^{\prime}\right)$ and $\phi^{*} \widehat{T}^{\prime}=-\omega_{\theta} \otimes \xi^{\prime}$. We deduce that any harmonic map $\phi: M \rightarrow N$ satisfies $\delta^{\nabla^{\prime}} d \phi=J^{\prime} d \phi(\xi)$ and $\left|\delta^{\nabla^{\prime}} d \phi\right|^{2}=\left|\xi^{\prime}-d \phi(\xi)\right|^{2}$. Now, since $i(\xi)\left(\phi^{*} \widehat{T}^{\prime}\right)=0$ and $\left(\phi^{*} \widehat{T}^{\prime}\right)_{H}^{J}=0$, Equations (14) and (15) yield:

$$
\begin{aligned}
& \int_{M}\left|\nabla_{\xi}(d \phi)_{H}\right|^{2}+\frac{1}{2}\left|\left(\nabla_{\xi} d \phi\right)(\xi)\right|^{2}+\frac{1}{2}\left|\delta^{\widehat{\nabla}^{\prime}}(d \phi)_{H}\right|^{2} \\
& -\frac{1}{2}\left|\xi^{\prime}-d \phi(\xi)\right|^{2} v_{g_{\theta}}=\int_{M}\left(\phi^{*} \widehat{R}^{\prime}\right)_{\xi} v_{g_{\theta}}, \\
& \int_{M}\left|d_{H}^{\widehat{\nabla}^{\prime}} J d \phi\right|^{2}-\left|\delta^{\widehat{\nabla}^{\prime}}(d \phi)_{H}\right|^{2}+d(d-1)\left|\xi^{\prime}-d \phi(\xi)\right|^{2} v_{g_{\theta}} \\
& =4 \int_{M}\left(\phi^{*} \hat{\rho}_{\mathbb{C}}^{\prime}\right)_{H}^{-} v_{g_{\theta}},
\end{aligned}
$$

Since $N$ has a strongly negative curvature, the Tanaka-Webster sectional curvature is nonpositive, hence $\left(\phi^{*} \widehat{R}^{\prime}\right)_{\xi}$ is nonpositive. Using (33), we deduce the following inequality:

$$
\int_{M}\left|\delta^{\widehat{\nabla}^{\prime}}(d \phi)_{H}\right|^{2} v_{g_{\theta}} \leq \int_{M}\left|\xi^{\prime}-d \phi(\xi)\right|^{2} v_{g_{\theta}} .
$$

Substituting it in (34), we obtain

$$
\int_{M}\left|d_{H}^{\widehat{\nabla}^{\prime}} J d \phi\right|^{2}+\left(d^{2}-d-1\right)\left|\xi^{\prime}-d \phi(\xi)\right|^{2} v_{g_{\theta}} \leq 4 \int_{M}\left(\phi^{*} \widehat{\rho}_{\mathbb{C}}^{\prime}\right)_{H}^{-} v_{g_{\theta}} .
$$

Since $N$ has a strongly negative curvature and $d \geq 2$, the previous inequality yields $d \phi(\xi)=\xi^{\prime}$ and $\left(\phi^{*} \widehat{\rho}_{\mathbb{C}}^{\prime}\right)_{H}^{-}=0$. The assumptions on the curvature and on the rank of $\phi$ imply using a similar proof as in Theorem 4.1 of [9] that $J^{\prime} \circ d \phi=d \phi \circ J$ or $J^{\prime} \circ d \phi=-d \phi \circ J$. Using $\phi^{*} \theta^{\prime}=\theta$, we deduce that necessarily $J^{\prime} \circ d \phi=d \phi \circ J$. Hence, $\phi$ is $C R$-holomorphic and, by Theorem 2.1 of [12], is an isometric immersion.

\section{Minimal isometric immersions and pseudo-Hermitian immersions.}

The following formulas for isometric immersions are the local analogues of (14) and (15). 
Proposition 6.1. Let $M$ be a strictly pseudoconvex $C R$ manifold and $N$ be a Riemannian manifold endowed with its Levi-Civita connection. For any isometric immersion $\phi: M \rightarrow N$ we have:

(35)

$\left|\nabla_{\xi}(d \phi)_{H}\right|^{2}+\frac{1}{2}\left|\left(\nabla_{\xi} d \phi\right)(\xi)\right|^{2}+\frac{1}{2}\left|\delta^{\hat{\nabla}^{\prime}}(d \phi)_{H}\right|^{2}-\frac{1}{2}\left|\delta^{\hat{\nabla}^{\prime}} d \phi\right|^{2}-|\tau|^{2}=\left(\phi^{*} \widehat{R}^{\prime}\right)_{\xi}$

and

$$
\left|d_{H}^{\widehat{\nabla}^{\prime}} J d \phi\right|^{2}-\left|\delta^{\hat{\nabla}^{\prime}}(d \phi)_{H}\right|^{2}+d(d-1)=4\left(\phi^{*} \widehat{\rho}_{\mathbb{C}}^{\prime}\right)_{H}^{-} .
$$

Lemma 6.1. Let $\phi$ be an isometric immersion, then, for any $X, Y, Z \in$ $T M$ :

$$
\begin{aligned}
\left\langle Y . \nabla_{X} d \phi, Z . d \phi\right\rangle= & \omega_{\theta}(X, Z) \theta(Y)+\omega_{\theta}(Y, Z) \theta(X)-\omega_{\theta}(X, Y) \theta(Z) \\
& +2\left(\theta(Z) A_{\theta}(X, Y)-\theta(Y) A_{\theta}(X, Z)\right)
\end{aligned}
$$

Proof. For any map $\phi: M \rightarrow N$ and any $X, Y, Z \in T M$, we have

$$
\left(\nabla_{X} \phi^{*} g^{\prime}\right)(Y, Z)=\left\langle\left(\nabla_{X} d \phi\right)(Y), d \phi(Z)\right\rangle+\left\langle d \phi(Y),\left(\nabla_{X} d \phi\right)(Z)\right\rangle .
$$

If $\phi$ is an isometric immersion, we have $\phi^{*} g^{\prime}=g_{\theta}$. Hence, $\nabla \phi^{*} g^{\prime}=0$ and $\left\langle\left(\nabla_{X} d \phi\right)(Z), d \phi(Y)\right\rangle=-\left\langle\left(\nabla_{X} d \phi\right)(Y), d \phi(Z)\right\rangle$. Using both (17) and the previous equality, we obtain

$$
\begin{aligned}
\left\langle Y . \nabla_{X} d \phi, Z . d \phi\right\rangle= & g_{\theta}(Y, Z)\left\langle\nabla_{X} d \phi, d \phi\right\rangle+\left\langle\left(\nabla_{X} d \phi\right)(Y), d \phi(Z)\right\rangle \\
& -\left\langle\left(\nabla_{X} d \phi\right)(Z), d \phi(Y)\right\rangle \\
= & 2\left\langle\left(\nabla_{X} d \phi\right)(Y), d \phi(Z)\right\rangle .
\end{aligned}
$$

Now, using (29), we have

$$
\begin{aligned}
&\left\langle\left(\nabla_{X} d \phi\right)(Y), d \phi(Z)\right\rangle \\
&= \frac{1}{2}\left(\theta(X) \omega_{\theta}(Y, Z)+\theta(Y) \omega_{\theta}(X, Z)\right) \\
&+\left(A_{\theta}(X, Y)-\frac{1}{2} \omega_{\theta}(X, Y)\right) \theta(Z)-\theta(Y) A_{\theta}(X, Z) \\
&= \frac{1}{2}\left(\omega_{\theta}(X, Z) \theta(Y)+\omega_{\theta}(Y, Z) \theta(X)-\omega_{\theta}(X, Y) \theta(Z)\right) \\
&+\theta(Z) A_{\theta}(X, Y)-\theta(Y) A_{\theta}(X, Z) .
\end{aligned}
$$

Hence the result. 
Proof of Proposition 6.1. Let $\phi$ be an isometric immersion. First, since $|d \phi|^{2}=m$, we have $\left\langle\nabla_{\xi, \xi}^{2} d \phi, d \phi\right\rangle=-\left|\nabla_{\xi} d \phi\right|^{2}$. Now, following the proof of Proposition 2.3, we have

$$
\begin{aligned}
\left\langle\mathcal{D}^{2} d \phi, d \phi\right\rangle-|\mathcal{D}(d \phi)|^{2} & =\delta \alpha, \\
\left\langle\mathcal{D}_{J}^{2}(d \phi), d \phi\right\rangle-\left|\mathcal{D}_{J}(d \phi)\right|^{2} & =\delta \beta, \\
\left\langle\mathcal{D}^{2}(\xi . d \phi), \xi . d \phi\right\rangle-|\mathcal{D}(\xi . d \phi)|^{2} & =\delta \gamma,
\end{aligned}
$$

where $\alpha, \beta$ and $\gamma$ are the 1 -forms respectively given by $\alpha(X)=\langle\mathcal{D} d \phi, X . d \phi\rangle$, $\beta(X)=\left\langle\mathcal{D}_{J}(d \phi), J X . d \phi\right\rangle$ and $\gamma(X)=\langle\mathcal{D}(\xi . d \phi), X . \xi . d \phi\rangle$. It follows from (37) that $\alpha$ and $\beta$ are zero. Using (2) and (37), we obtain

$$
\begin{aligned}
\gamma(X) & =\langle\mathcal{D}(\xi . d \phi), X . \xi . d \phi\rangle=-\langle\xi . \mathcal{D}(d \phi), X . \xi . d \phi\rangle-2\left\langle\nabla_{\xi} d \phi, X . \xi . d \phi\right\rangle \\
& =\langle\mathcal{D}(d \phi), X . d \phi\rangle-2 \theta(X)\langle\mathcal{D}(d \phi), \xi . d \phi\rangle+2\left\langle X . \nabla_{\xi} d \phi, \xi . d \phi\right\rangle \\
& =0
\end{aligned}
$$

Always using (37), we have $\left\langle\mathcal{D}_{\tau} d \phi, \xi . d \phi\right\rangle=2|\tau|^{2}$. Now, by noting that $\langle d \phi \circ J \circ \tau, d \phi\rangle=-\left\langle A_{\theta}, \omega_{\theta}\right\rangle=0$ and $\langle d \phi(\operatorname{Ric}(\xi)), d \phi(\xi)\rangle=0$, we conclude the proof as in propositions 3.1 and 3.2 using Lemma 3.1 and Lemma 3.3.

Remember that a harmonic isometric immersion is said to be a minimal isometric immersion. The following Theorem holds

Theorem 6.1. Let $M$ be a strictly pseudoconvex $C R$ manifold of dimension $2 d+1$ and $N$ be a Riemannian manifold with nonpositive complex sectional curvature. If $|\tau|^{2} \leq d(d-1)$ at some point, then there is no minimal isometric immersion from $M$ to $N$.

Proof. First, suppose that $d>1$. If $\phi: M \rightarrow N$ is a minimal isometric immersion, then $\delta^{\prime}(d \phi)_{H}=\left(\nabla_{\xi} d \phi\right)(\xi)$. Hence, adding (35) and (36), we obtain

$$
\left|\nabla_{\xi}(d \phi)_{H}\right|^{2}+\left|d_{H}^{\hat{\nabla}^{\prime}} J d \phi\right|^{2}+d(d-1)-|\tau|^{2}=\left(\phi^{*} \widehat{R}^{\prime}\right)_{\xi}+4\left(\phi^{*} \widehat{\rho}_{\mathbb{C}}^{\prime}\right)_{H}^{-} .
$$

Now, at a point where $|\tau|^{2} \leq d(d-1)$, we deduce, using the previous equation and the curvature assumption, that $|\tau|^{2}=d(d-1)$ and $\nabla_{\xi}(d \phi)_{H}=0$. In this case, Equation (13) gives for any $X \in H$

$$
\left(\nabla_{X} d \phi\right)(\xi)=-(d \phi \circ \tau)(X) .
$$


Now, on the one hand, we have for any $X, Y \in H$

$$
\left\langle\left(\nabla_{X} d \phi\right)(\xi), d \phi(\tau(Y))\right\rangle=-g_{\theta}(\tau(X), \tau(Y)) .
$$

On the other hand, using (37), we have

$$
\left\langle\left(\nabla_{X} d \phi\right)(\xi), d \phi(\tau(Y))\right\rangle=\frac{1}{2} \omega_{\theta}(X, \tau(Y))-g_{\theta}(\tau(X), \tau(Y)) .
$$

We deduce that $\omega_{\theta}(X, \tau(Y))=0$ for any $X, Y \in H$, and consequently, $\tau(Y)=0$ (because $\tau(Y) \in H)$. Hence $\tau=0$ at this point, which contradicts $|\tau|^{2}=d(d-1)$. In the case $d=1, M$ is assumed to be a Sasakian manifold, hence following the proof of Theorem 4.1, the result holds too.

Theorem 6.2. Let $M$ be a $2 d+1$-dimensional strictly pseudoconvex $C R$ manifold $(2 d+1>3)$ endowed with its Webster metric and with the associated Levi-Civita connection. At each point of $M$, there exists a complex 2-plane for which the complex sectional curvature is positive.

Proof. Since the identity map $I$ is a minimal isometric immersion, we obtain using (36):

$$
\left|d_{H}^{D} J I\right|^{2}+d(d-1)=4\left(\widehat{\rho}_{\mathbb{C}}\right)_{H}^{-} .
$$

Now, for any $X, Y \in T M$, we have by (29):

$$
\left(\nabla_{J X} I\right)(Y)=-\frac{1}{2} \theta(Y) X+\frac{1}{2} g_{\theta}(X, Y) \xi-\omega_{\theta}(\tau(X), Y) \xi+\theta(Y)(J \circ \tau)(X)
$$

Hence, for any $X, Y \in H$,

$$
\left(d_{H}^{D} J I\right)(X, Y)=\left(d^{D} J I\right)(X, Y)=\left(\nabla_{J X} I\right)(Y)-\left(\nabla_{J Y} I\right)(X)=0 .
$$

We deduce that:

$$
d(d-1)=4\left(\widehat{\rho}_{\mathbb{C}}\right)_{H}^{-} .
$$

The theorem is directly deduced from this last equation.

Remark 6.1. Applying (35) to the identity map, we recover the relation between the Ricci curvature and the pseudo-Hermitian torsion obtained by Rumin ([17]). 
A nonexistence result for pseudo-Hermitian immersions.

In this subsection $N$ is a Sasakian manifold endowed with its TanakaWebster connection $\nabla^{\prime}$. A pseudo-Hermitian immersion $\phi: M \rightarrow N$ is an isometric (with respect to the Webster metrics) and a $C R$-holomorphic immersion. Note that a pseudo-Hermitian immersion satisfies $\phi^{*} \theta^{\prime}=\theta$ and $d \phi(\xi)=\xi^{\prime}$.

Theorem 6.3. Let $M$ be a non-Sasakian strictly pseudoconvex $C R$ manifold. Then, there is no pseudo-Hermitian immersion between $M$ and $N$.

As a corollary, we obtain (cf. [1]):

Corollary 6.1. There is no pseudo-Hermitian immersion from $\mathcal{H}^{d}(s)$ into Pseudo-Siegel domains.

Proof of Theorem 6.3. Suppose that there exists a pseudo-Hermitian immersion $\phi: M \rightarrow N$. Then, since $\phi$ is pseudo-Hermitian, we have $\left(\nabla_{X} d \phi\right)(\xi)=\nabla_{X}^{\prime} \xi^{\prime}=0$, for any $X \in T M$. Since $\phi^{*} \widehat{T}^{\prime}=-\omega_{\theta} \otimes d \phi(\xi)$, we deduce from (13) that

$$
\nabla_{\xi} d \phi=d \phi \circ \tau
$$

And consequently,

$$
\left\langle\nabla_{\xi} d \phi, d \phi \circ \tau\right\rangle=|\tau|^{2} .
$$

On the other hand, since $\phi$ is a pseudo-Hermitian immersion, we have $\phi^{*} \widehat{U}^{\prime}=$ $-\frac{1}{2}\left(\theta \odot d \phi \circ J-\omega_{\theta} \otimes d \phi(\xi)\right)$. Now, we have from (29):

$$
\left\langle\nabla_{\xi} d \phi, d \phi \circ \tau\right\rangle=\left\langle D_{\xi} d \phi, d \phi \circ \tau\right\rangle=0 .
$$

Hence $\tau=0$, contradicting the assumption on $M$.

\section{References.}

[1] E. Barletta and S. Dragomir, Pseudohermitian immersions, PseudoEinstein structures, and the Lee class of a CR manifold, Kodai. Math. J., 19 (1996), 62-86.

[2] D.E. Blair, Contact manifolds in Riemannian Geometry, Lecture Notes in Math., 509, Springer-Verlag, New York, 1976. 
[3] J.A. Carlson and D. Toledo, Harmonic maps of Kähler manifolds to locally symmetric spaces, Publ. Maths. IHES., 69 (1989), 173-201.

[4] K. Corlette, Archimedian superrigidity and hyperbolic geometry, Ann. of Math., 135 (1992), 165-182.

[5] K. Corlette, Flat G-bundles with canonical metrics, J. Differential Geom., 28 (1988), 361-382.

[6] S. Dragomir, On pseudo-Hermitian immersions between strictly pseudoconvex CR manifolds, Amer. J. of Math., 117 (1995), 169-202.

[7] S. Dragomir, On a conjecture of J.M. Lee, Hokkaido. Math. J., 23 (1994), 35-49.

[8] J. Eells and J.H. Sampson, Harmonic mappings of Riemannian manifolds, Amer. J: Math., 86 (1964), 109-160.

[9] A. El Soufi and R. Petit, Applications harmoniques, applications pluriharmoniques et existence de 2-formes parallèles non nulles, Comment. Math. Helvetici., 73 (1998), 1-21.

[10] E. Falbel and C. Gorodski, On contact sub-Riemannian symmetric spaces, Ann. scient. Ec. Norm. Sup., 28 (1995), 571-589.

[11] L. Hernandez, Kähler manifolds and 1/4 Pinching, Duke Math. J., 62 (1991), 601-611.

[12] S. Ianus and A.M. Pastore, Harmonic maps on contact metric manifolds, Ann. Math. Blaise Pascal., 2 (1995), 43-53.

[13] S. Ianus and A.M. Pastore, Harmonic maps on Sasakian and cosymplectic manifolds, in 'Proceedings of the conference on Geometry and Topology,' Timisoara, Editura Mirton, (1996), 139-155.

[14] H.B. Lawson and M.L. Michelsohn, Spin Geometry, Princeton Math. Ser. 38, Princeton University Press, Princeton, 1989.

[15] J. Lee, Pseudo-Einstein structures on CR manifolds, Amer. J. of Math., 110 (1988), 157-178.

[16] N. Mok, Y.T. Siu and S.K. Yeung, Geometric superrigidity, Invent. Math., 113 (1993), 57-83. 
[17] M. Rumin, Formes différentielles sur les variétés de contact, J. Differential Geom., 39 (1994), 281-330.

[18] M. Rumin, Sub-Riemannian limit of the differential form spectrum of contact manifolds, Geo. An. Func. Appl., 10 (2000), 407-452.

[19] J.H. Sampson, Harmonic maps in Kähler geometry, CIME Conf. - 1984, Lecture Notes in Math., 1161, Springer-Verlag, New York, (1985), 193205.

[20] Y.T. Siu, The complex analyticity of harmonic maps and the strong rigidity of compact Kähler manifolds, Ann. of Math., 112 (1980), 73111.

[21] N. Tanaka, A Differential Geometric Study on strongly pseudoconvex CR manifolds, Lecture Notes in Math., 9, Kyoto University, 1975.

[22] S. Tanno, The standard CR structure on the unit tangent bundle, Tohoku. Math. J., 44 (1992), 535-543.

[23] H. Urakawa, Yang-Mills connections over compact strongly pseudoconvex CR manifolds, Math. Z., 216 (1994), 541-573.

[24] H. Urakawa, Variational problems over strongly pseudoconvex $C R$ manifolds, Differential Geometry, Editors C.H. Gu, H.S. Ho and Y.L. Xin, World scientific, Singapore-New Jersey-London-Hong Kong, (1993), 233-242.

[25] S. Webster, Pseudo-Hermitian structures on a real hypersurface, J. Differential Geom., 13 (1978), 25-41.

UMR 6629 CNRS

UNIVERSITÉ DE NANTES

DÉPARTEMENT DE MATHÉMATIQUeS

2, RUE DE LA HoussiniÈre BP 92208

44322 NANTES - FRANCE

E-mail address: petit@math.univ-nantes.fr

Received August 29, 2000. 